\title{
$\mathrm{J} / \psi$ production as a function of charged-particle multiplicity in $\mathrm{p}-\mathrm{Pb}$ collisions at $\sqrt{s_{\mathrm{NN}}}=8.16 \mathrm{TeV}$
}

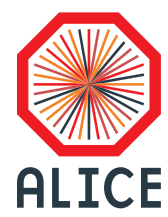

\section{The ALICE collaboration}

\section{E-mail: ALICE-publications@cern.ch}

ABSTRACT: Inclusive $\mathrm{J} / \psi$ yields and average transverse momenta in $\mathrm{p}-\mathrm{Pb}$ collisions at a center-of-mass energy per nucleon pair $\sqrt{s_{\mathrm{NN}}}=8.16 \mathrm{TeV}$ are measured as a function of the charged-particle pseudorapidity density with ALICE. The $\mathrm{J} / \psi$ mesons are reconstructed at forward $\left(2.03<y_{\mathrm{cms}}<3.53\right)$ and backward $\left(-4.46<y_{\mathrm{cms}}<-2.96\right)$ center-of-mass rapidity in their dimuon decay channel while the charged-particle pseudorapidity density is measured around midrapidity. The $\mathrm{J} / \psi$ yields at forward and backward rapidity normalized to their respective average values increase with the normalized charged-particle pseudorapidity density, the former showing a weaker increase than the latter. The normalized average transverse momenta at forward and backward rapidity manifest a steady increase from low to high charged-particle pseudorapidity density with a saturation beyond the average value.

KEYWORDS: Hadron-Hadron scattering (experiments), Particle correlations and fluctuations

ARXIV EPRINT: 2004.12673 


\section{Contents}

1 Introduction 1

2 Experimental setup and data samples 2

3 Charged-particle multiplicity measurement 3

$4 \mathrm{~J} / \psi$ measurement $\quad 4$

$\begin{array}{lll}5 & \text { Systematic uncertainties } & 7\end{array}$

6 Results and discussion $\quad 9$

$\begin{array}{lll}7 & \text { Conclusions } & 14\end{array}$

The ALICE collaboration $\quad 22$

\section{Introduction}

Quarkonium states have long been considered as probes of the Quark-Gluon Plasma (QGP) produced in ultra-relativistic heavy-ion collisions [1]. The large color-charge density in the plasma prevents the formation of bound states, in an analogous process to the Debye screening for electromagnetic processes [2]. The suppression of $\mathrm{J} / \psi$ production in nucleus-nucleus (AA) with respect to proton-proton (pp) collisions was observed by several experiments [3-11]. To determine whether the origin of this suppression is the influence of the QGP or of Cold Nuclear Matter (CNM), data on proton(deuteron)-nucleus collisions are also scrutinized.

The measurements in $\mathrm{p}-\mathrm{Pb}$ collisions at the LHC show a suppression of $\mathrm{J} / \psi$ production [12-14], with respect to pp collisions, at low transverse momentum $\left(p_{\mathrm{T}}\right)$ and forward center-of-mass rapidity (p-going direction, positive $y_{\mathrm{cms}}$ ), consistent with various combinations of CNM effects: modification of the parton distribution functions (PDFs) in nuclei, i.e. shadowing $[15,16]$, the Color-Glass Condensate (CGC) $[17,18]$, or coherent parton energy loss [19]. The measurement of $\psi(2 \mathrm{~S})$ production in $\mathrm{p}-\mathrm{Pb}$ collisions [20] exhibits a larger suppression, with respect to pp collisions, than the one measured for $\mathrm{J} / \psi$, both at forward and backward rapidity, which was not expected from CNM predictions. This effect is reproduced by models which consider the break-up of the bound quark-anti-quark pair via interactions with the final-state comoving particles [21, 22].

The p- $\mathrm{Pb}$ data at the center-of-mass energy per nucleon-nucleon collision of $\sqrt{s_{\mathrm{NN}}}=$ $5.02 \mathrm{TeV}[23,24]$ showed that these effects depend on the centrality of the collision, as 
estimated from the energy deposited in the Zero Degree Calorimeter in the Pb-going direction [25], and/or the produced charged-particle multiplicity [26]. An increase of the normalized $\mathrm{J} / \psi$ and $\Upsilon[26-28]$ yields, to their respective average values, with the normalized charged-particle multiplicity is observed, similarly to the results from pp collisions [27-29]. The increase of the $\mathrm{J} / \psi$ (prompt and non-prompt) normalized yields was observed to be similar to the increase for D mesons [30,31], suggesting that the origin of the trend is common for charm and beauty production, and that hadronization does not play a dominant influence on this measurement. The excited-to-ground state ratios, $\Upsilon(\mathrm{nS}) / \Upsilon(1 S)$, were found to decrease with increasing charged-particle multiplicity, which was not expected from CNM predictions [27, 28].

The measurements of two-particle angular correlations in small systems have shown interesting structures in the angular correlation function. A near-side ridge, located at $(\Delta \varphi) \approx 0$, is observed in high-multiplicity pp [32] and p- $\mathrm{Pb}$ [33] collisions, accompanied by an away-side structure, located at $\Delta \varphi \approx \pi$ and exceeding the away-side jet contribution, in $\mathrm{p}-\mathrm{Pb}$ collisions [34, 35]. These structures are reminiscent of those in $\mathrm{Pb}-\mathrm{Pb}$ data [36], interpreted as signatures of the collective motion of the particles during the hydrodynamic evolution of the hot and dense medium. Correlations of $\mathrm{J} / \psi$ (at large rapidity) and charged particles (at midrapidity) in p- $\mathrm{Pb}$ collisions [37, 38] revealed persisting long-range correlation structures at high $p_{\mathrm{T}}$, similar to those observed with charged hadrons. The corresponding elliptic flow coefficients are found to be positive and of comparable magnitude to those measured in $\mathrm{Pb}-\mathrm{Pb}$ collisions [39-41], indicating that the mechanism at its origin could be similar in both collision systems.

This letter reports the measurement of the multiplicity-differential inclusive $\mathrm{J} / \psi$ yield and average transverse momentum in $\mathrm{p}-\mathrm{Pb}$ collisions at $\sqrt{s_{\mathrm{NN}}}=8.16 \mathrm{TeV}$. The $\mathrm{J} / \psi$ mesons are reconstructed at forward and backward center-of-mass rapidities in their dimuon decay channel. The charged-particle pseudorapidity density is measured around midrapidity. It complements and extends previous $\mathrm{J} / \psi$ measurements performed as a function of the collision centrality and the charged-particle multiplicity at $\sqrt{s_{\mathrm{NN}}}=5.02 \mathrm{TeV}[23,26]$. The classification of events as a function of their charged-particle pseudorapidity density enables the scrutiny of rare events, corresponding to the $0.01-0.04 \%$ highest multiplicities in the collision. This allows p- $\mathrm{Pb}$ events to be studied from low multiplicities, similar to those of pp collisions, up to very large multiplicities corresponding to $\sim 100$ produced charged particles per rapidity unit, similar to those of peripheral $\mathrm{Pb}-\mathrm{Pb}$ collisions, which exhibit collective-like effects.

\section{Experimental setup and data samples}

In this section, the detector subsystems relevant for this analysis are presented. A complete description of the ALICE detector and its performance can be found in [42, 43].

The muon spectrometer $[42,43]$ covers the pseudorapidity window of $-4.0<\eta<-2.5$ and consists of: a $4 \mathrm{~m}$ long composite front absorber, corresponding to about 10 interaction lengths $\left(10 \lambda_{\text {int }}\right)$, starting at $90 \mathrm{~cm}$ from the nominal interaction point, ten layers of muon tracking chambers $(\mathrm{MCH})$, coupled to a dipole magnet with a $3 \mathrm{Tm}$ field integral, and 
four layers of muon trigger chambers (MTR). The MCH and MTR systems are separated by an additional iron wall of about 7.2 $\lambda_{\text {int }}$ that absorbs the remaining hadronic and lowmomentum particle contamination. A rear absorber positioned downstream of the MTR filters out the background from beam-gas interactions. A conical absorber surrounds the beam pipe and protects the spectrometer against secondary particles produced mainly by large- $\eta$ primary particles interacting with the beam pipe.

The Silicon Pixel Detector (SPD) [44] is the innermost part of the Inner Tracking System (ITS). It consists of two cylindrical silicon pixel layers at radial distances of 3.9 and $7.6 \mathrm{~cm}$ from the beam line. The respective pseudorapidity coverage of the two layers are $|\eta|<2$ and $|\eta|<1$.4. The SPD is used to reconstruct the primary vertex and to measure the charged-particle pseudorapidity density at midrapidity.

The V0 scintillator arrays [45] are located at each side of the interaction point, covering the pseudorapidity ranges of $-3.7<\eta<-1.7$ and $2.8<\eta<5.1$. In this analysis, the V0 provides an online trigger and helps to reject contamination from beam-gas events.

The neutron Zero Degree Calorimeter (ZDC) [42] located at about $112.5 \mathrm{~m}$ on either side from the interaction point are used to reject electromagnetic interactions and beaminduced background.

The results presented in this letter are obtained with data recorded during the $\mathrm{p}-\mathrm{Pb}$ run at $\sqrt{s_{\mathrm{NN}}}=8.16 \mathrm{TeV}$ in 2016. The $\mathrm{J} / \psi$ are reconstructed in the dimuon channel with data taken in two different beam configurations. Due to the asymmetry of the beam energy per nucleon in $\mathrm{p}-\mathrm{Pb}$ collisions at the $\mathrm{LHC}$, the nucleon-nucleon center-of-mass rapidity frame is shifted by $\Delta y=0.465$ in the direction of the proton beam. As a consequence, the $\mathrm{J} / \psi$ are measured in the forward rapidity range of $2.03<y_{\mathrm{cms}}<3.53$ (with protons going in the direction of the muon spectrometer, p-going direction) and in the backward rapidity region $-4.46<y_{\mathrm{cms}}<-2.96$ (Pb-going direction). Events used in this analysis were collected with a dedicated dimuon trigger which requires the coincidence of signals in both V0 arrays (minimum bias trigger, MB) with at least two opposite-sign muons registered in the MTR. The trigger has an adjustable online threshold, which for this data sample was set to only accept muons with transverse momenta $p_{\mathrm{T}}>0.5 \mathrm{GeV} / c$ ( $p_{\mathrm{T}}$ for which an efficiency of $50 \%$ is reached). The $p_{\mathrm{T}}$ differential single-muon trigger efficiency reaches a plateau of $\sim 96 \%$ at $p_{\mathrm{T}} \sim 1.5 \mathrm{GeV} / c$. In this data-taking period, the maximum pile-up probability was about 4\%. A dedicated event-selection strategy - exploiting the signals in the V0 and the ZDC, the correlation of the number of clusters and track segments reconstructed in the SPD, as well as an algorithm to tag events with multiple vertices - allowed us to keep the pile-up below $0.5 \%$ for the analysed events, even at large multiplicities. The data sample analyzed corresponds to an integrated luminosity of $\mathcal{L}_{\text {int }}=7.2 \pm 0.2 \mathrm{nb}^{-1}\left(10.6 \pm 0.3 \mathrm{nb}^{-1}\right)$ for the p-going (Pb-going) configuration [46].

\section{Charged-particle multiplicity measurement}

The charged-particle pseudorapidity density $\left(\mathrm{d} N_{\mathrm{ch}} / \mathrm{d} \eta\right)$ is measured at midrapidity exploiting the information provided by the SPD detector [47, 48]. It is evaluated by counting the number of tracklets $\left(N_{\text {tracklet }}\right)$, i.e. track segments joining pairs of hits in the two layers of the SPD pointing to the primary vertex. The primary vertex is also computed with 


\begin{tabular}{|c|c|}
\hline Source & $|\eta|<1$ \\
\hline$N_{\text {tracklet }}^{\text {corr }}$ to $\mathrm{d} N_{\mathrm{ch}} / \mathrm{d} \eta$ correlation & $0.1-6.9(5.8) \%$ \\
$z$-vertex dependence & $3 \%$ \\
Monte Carlo event generator & $2 \%$ \\
$\left\langle\mathrm{~d} N_{\mathrm{ch}} / \mathrm{d} \eta\right\rangle$ & $4 \%$ \\
\hline
\end{tabular}

Table 1. Sources of systematic uncertainties on the normalized charged-particle multiplicity. For the $N_{\text {tracklet }}^{\text {corr }}$ to $\mathrm{d} N_{\mathrm{ch}} / \mathrm{d} \eta$ correlation an interval is quoted, varying with multiplicity, with a different maximum uncertainty for the $\mathrm{Pb}(\mathrm{p})$-going configuration.

the SPD information. To minimize non-uniformities in the SPD acceptance, only events with a $z$-vertex position determined within $\left|z_{\mathrm{vtx}}\right|<10 \mathrm{~cm}$ are considered, and tracklets are counted within $|\eta|<1$.

The raw $N_{\text {tracklet }}$ counts are corrected $\left(N_{\text {tracklet }}^{\text {corr }}\right)$ for the variation of the detector conditions with time (fraction of active SPD channels) and its limited acceptance as a function of $z_{\mathrm{vtx}}$ using a data-driven event-by-event correction [29,30]. This correction ensures a uniform response as a function of $z_{\mathrm{vtx}}$. In this analysis, the correction is done by renormalising the $N_{\text {tracklet }}\left(z_{\mathrm{vtx}}\right)$ distributions to the overall maximum with a Poissonian smearing to account for the fluctuations. The events are sliced in $N_{\text {tracklet }}^{\text {corr }}$ intervals. Monte Carlo (MC) simulations using the DPMJET [49] event generator and the GEANT3 transport code [50] are used to estimate $\mathrm{d} N_{\mathrm{ch}} / \mathrm{d} \eta$ from $N_{\text {tracklet. }}^{\text {corr }}$. A second order polynomial correlation is assumed between these two quantities for the full $N_{\text {tracklet }}^{\text {corr }}$ interval. Several sources of systematic uncertainty were taken into account. Possible deviations from the second order polynomial correlation were estimated by using other functions to quantify the correlation or $\mathrm{MC}$ averages in each interval, with values ranging from $0.1 \%$ at intermediate multiplicities to $6.9 \%(5.8 \%)$ at the lowest (highest) multiplicity intervals. The systematic uncertainty on the residual $z_{\mathrm{vtx}}$ dependence due to differences between data and MC amounts to $3 \%$. Finally, the event generator influence was considered and evaluated by comparing the DPMJET simulations with events generated in EPOS [51], resulting in a $2 \%$ uncertainty.

The average charged-particle pseudorapidity density, $\left\langle\mathrm{d} N_{\mathrm{ch}} / \mathrm{d} \eta\right\rangle$, in non-single diffractive (NSD) events was obtained from an independent analysis and amounts to $\left\langle\mathrm{d} N_{\mathrm{ch}} / \mathrm{d} \eta\right\rangle=$ $20.33 \pm 0.83(20.32 \pm 0.83)$ in $\mathrm{p}-\mathrm{Pb}(\mathrm{Pb}-\mathrm{p})$ collisions for $|\eta|<1$ [48], where the quoted uncertainty is systematic.

Table 1 summarizes the contributions to the normalized charged-particle multiplicity uncertainty. The total uncertainty is evaluated assuming that the different sources are uncorrelated.

\section{$4 \mathrm{~J} / \psi$ measurement}

The normalized $\mathrm{J} / \psi$ yield, i.e. the yield in each multiplicity interval $i$ normalized to the multiplicity-integrated value, is evaluated as

$$
\frac{\mathrm{d} N^{i} / \mathrm{d} y}{\langle\mathrm{~d} N / \mathrm{d} y\rangle}=\frac{N_{\mathrm{J} / \psi}^{i}}{N_{\mathrm{J} / \psi}} \frac{N_{\mathrm{MB}}^{\mathrm{eq}}}{N_{\mathrm{MB}}^{i, \mathrm{eq}}} \frac{(A \varepsilon)_{\mathrm{J} / \psi}}{(A \varepsilon)_{\mathrm{J} / \psi}^{i}} \frac{\varepsilon_{\mathrm{MB}}^{i}}{\varepsilon_{\mathrm{MB}}},
$$


from the reconstructed number of $\mathrm{J} / \psi, N_{\mathrm{J} / \psi}$, the number of minimum bias (MB) events equivalent to the analysed dimuon sample, $N_{\mathrm{MB}}^{\mathrm{eq}}$, the $\mathrm{J} / \psi$ acceptance and efficiency correction, $(A \varepsilon)_{\mathrm{J} / \psi}$, and the NSD event selection efficiency in the minimum bias sample, $\varepsilon_{\mathrm{MB}}$.

The $\mathrm{J} / \psi$ are reconstructed for each multiplicity interval by combining opposite-sign muons and computing the invariant mass of the pairs. The muon identification is ensured by requiring that the track candidates reconstructed in the $\mathrm{MCH}$ have a matching track segment in the MTR. Furthermore, the individual tracks must fulfill the following criteria to make sure they are within the acceptance of the spectrometer: their radial distance from the beam axis at the end of the front absorber is within $17.6<R_{\text {abs }}<89.5 \mathrm{~cm}$ and their pseudorapidity in the detector reference frame is within $-4<\eta<-2.5$.

To extract the signal, the invariant-mass distributions are first corrected for the $\mathrm{J} / \psi$ acceptance times efficiency $(A \varepsilon)$, differentially in $p_{\mathrm{T}}$ and $y$. The resulting distributions are then fitted with a superposition of $\mathrm{J} / \psi$ and $\psi(2 \mathrm{~S})$ signals and a background lineshape. Various combinations of lineshapes are used in order to evaluate the signal counts and their uncertainties. The two charmonium resonances are parametrized by a sum of either two Crystal Ball or two pseudo-Gaussian functions with power-law tails [52]. The tail parametrizations are fixed to the values determined from either fits of the $\mathrm{J} / \psi$ signal from $\mathrm{MC}$ simulations or to values taken from fits to the multiplicity-integrated distribution in $\mathrm{p}-\mathrm{Pb}$ data at $\sqrt{s_{\mathrm{NN}}}=8.16 \mathrm{TeV}[13]$ and in pp data at $\sqrt{s}=13 \mathrm{TeV}$ [53]. The tails obtained from fitting the multiplicity-integrated distributions using the Crystal Ball function are also considered, and fixed in the binned fits. The $\mathrm{J} / \psi$ peak mean position and width are left free in the multiplicity-integrated fit, whilst the $\psi(2 \mathrm{~S})$ ones are bound to those of the $\mathrm{J} / \psi$ following the same procedure as in [54]. Note that the $\psi(2 \mathrm{~S})$ yields obtained are not physical values, as the invariant-mass spectrum is corrected by the $A \varepsilon$ correction for the $\mathrm{J} / \psi$. In the multiplicity-differential fits, the mass and width of the $\mathrm{J} / \psi$ peak are fixed to the integrated values to ensure the convergence of the fits in the few cases where statistical significance is low. The background is parameterized by either a sum of two exponentials or the product of an exponential and a fourth-order polynomial. Two fit mass ranges are taken into account when computing the average number of $\mathrm{J} / \psi$ and its uncertainty: $1.7<m_{\mu \mu}<4.8 \mathrm{GeV} / c^{2}$ and $2.0<m_{\mu \mu}<5.0 \mathrm{GeV} / c^{2}$. Examples of fits at low, intermediate, and high multiplicity for data in the rapidity range $2.03<y_{\mathrm{cms}}<3.53$ are shown in figure 1 . The signal lineshape is found to be independent of multiplicity, while the background does change with multiplicity. Therefore, in order to minimize the uncertainty on the signal extraction, the same signal lineshape is used in the fit function for both the numerator and denominator in eq. 4.1.

The number of equivalent $\mathrm{MB}$ events $N_{\mathrm{MB}}^{\mathrm{eq}}$ is computed from the number of dimuon triggered events, $N_{\mu \mu}$, and the normalization factor of dimuon triggered to MB events (calculated as explained in next section) as $N_{\mathrm{MB}}^{\mathrm{eq}}=F_{\text {norm }} \cdot N_{\mu \mu}$. The number needs to be corrected for by the NSD event selection efficiency, $\varepsilon_{\mathrm{MB}}=(97 \pm 1) \%$ [48], to take into account the fraction of events without a reconstructed SPD vertex that are rejected. This factor $\varepsilon_{\mathrm{MB}}$ is found to be independent of the charged-particle multiplicity in all the intervals studied, with the exception of the lowest multiplicity interval, where it decreases by $1 \%$.

The $\mathrm{J} / \psi$ acceptance and efficiency correction is obtained from MC simulations as a function of $p_{\mathrm{T}}$ and $y_{\mathrm{cms}}$. The $\mathrm{J} / \psi$ are generated using $p_{\mathrm{T}}$ and $y_{\mathrm{cms}}$ distributions tuned 

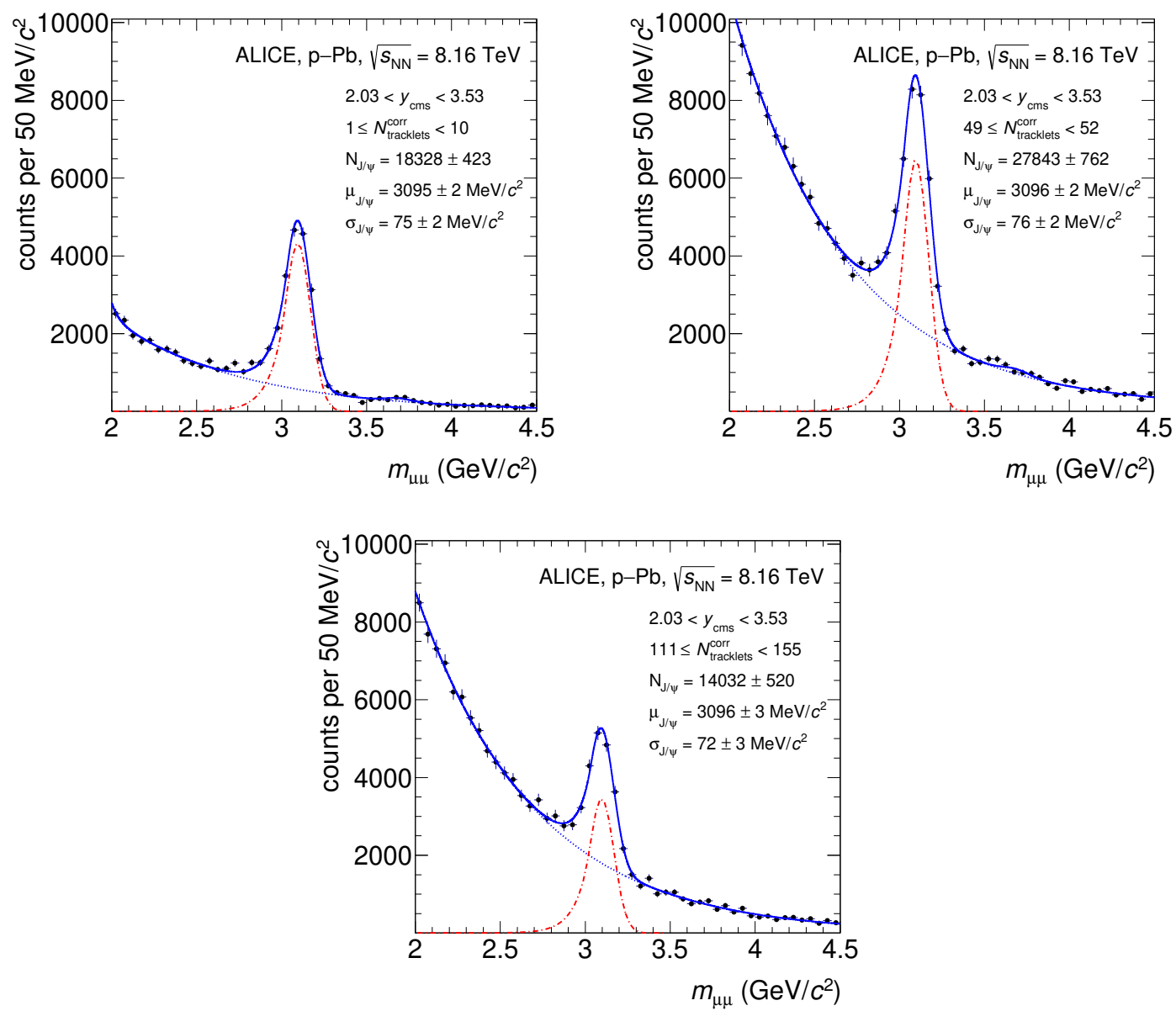

Figure 1. Opposite-sign muon pair invariant mass distributions for selected multiplicity intervals, corrected for the $\mathrm{J} / \psi$ acceptance and efficiency, at forward rapidity. The distributions are shown together with a typical fit function (solid line, see text for details). The $\mathrm{J} / \psi$ signal contribution is also depicted by a dot-dashed red line, and the background by a dotted line.

to data [13]. They are simulated to decay into a muon pair using EvtGen [55]. The final state radiation is described with PHOTOS [56]. The acceptance and efficiency correction is independent of multiplicity in the measurement intervals. Therefore, when estimating the uncertainty on the $\mathrm{MC}$ input, only the possible variation of the input $p_{\mathrm{T}}$ and $y_{\mathrm{cms}}$ distributions is taken into account by using as input a subsample of the lower/higher multiplicity events.

To extract the $\mathrm{J} / \psi$ mean transverse momentum $\left\langle p_{\mathrm{T}}^{\mathrm{J} / \psi}\right\rangle$, the $A \varepsilon$-corrected transverse momentum of the dimuon pair is fitted with the following function [26]:

$$
\begin{aligned}
\left\langle p_{\mathrm{T}}^{\mu \mu}\right\rangle\left(m_{\mu \mu}\right)= & \alpha^{\mathrm{J} / \psi}\left(m_{\mu \mu}\right)\left\langle p_{\mathrm{T}}^{\mathrm{J} / \psi}\right\rangle \\
& +\alpha^{\psi^{\prime}}\left(m_{\mu \mu}\right)\left\langle p_{\mathrm{T}}^{\psi^{\prime}}\right\rangle \\
& +\left(1-\alpha^{\mathrm{J} / \psi}\left(m_{\mu \mu}\right)-\alpha^{\psi^{\prime}}\left(m_{\mu \mu}\right)\right)\left\langle p_{\mathrm{T}}^{\mathrm{bkgd}}\right\rangle\left(m_{\mu \mu}\right),
\end{aligned}
$$

where the ratios of signal over the sum of signal and background of the two charmonium 

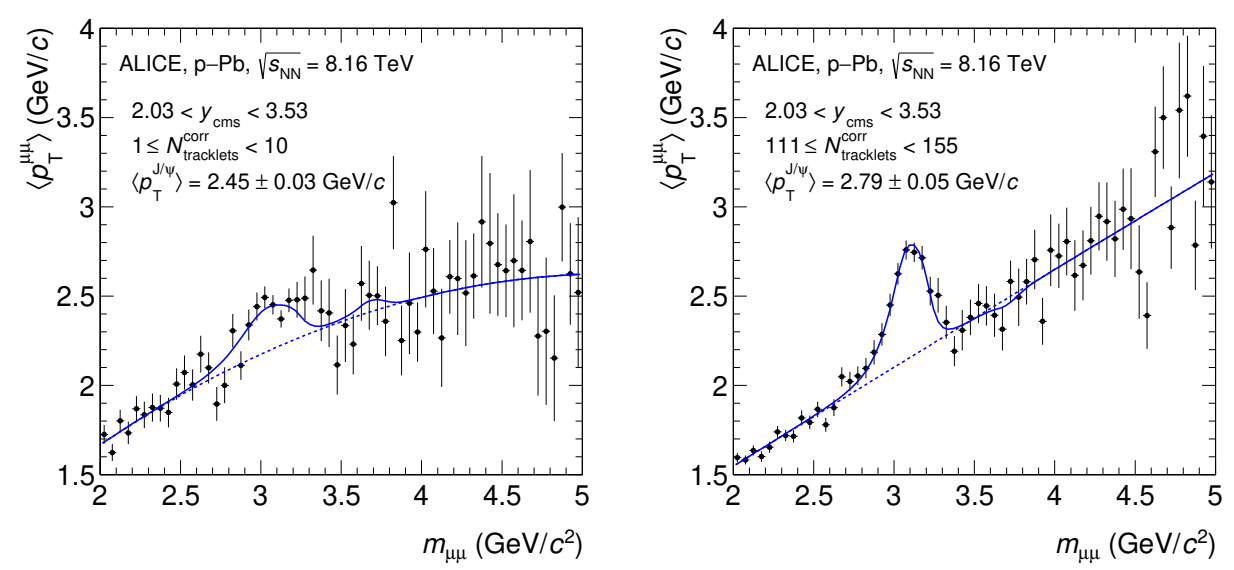

Figure 2. Average transverse momentum of opposite-sign muon pairs for selected multiplicity intervals, corrected for the $\mathrm{J} / \psi$ acceptance and efficiency. The distributions are shown together with a typical fit function (solid line, see text for details).

states $\alpha^{\mathrm{J} / \psi}=S^{\mathrm{J} / \psi} /\left(S^{\mathrm{J} / \psi}+S^{\psi^{\prime}}+B\right)$ and $\alpha^{\psi^{\prime}}=S^{\psi^{\prime}} /\left(S^{\mathrm{J} / \psi}+S^{\psi^{\prime}}+B\right)$ are fixed to the value extracted from fitting the invariant-mass spectrum corrected by the $\mathrm{J} / \psi A \varepsilon$. The background is described by a function $\left\langle p_{\mathrm{T}}^{\mathrm{bkgd}}\right\rangle\left(m_{\mu \mu}\right)$. Two functional forms are used: either a sum of two exponentials or the product of an exponential and a fourth-order polynomial. Note that the $\left\langle p_{\mathrm{T}}^{\psi^{\prime}}\right\rangle$ does not represent a physical mean transverse momentum of the $\psi(2 \mathrm{~S})$ as the spectra are corrected by the $A \varepsilon$ for $\mathrm{J} / \psi$. Figure 2 illustrates typical $\left\langle p_{\mathrm{T}}^{\mu \mu}\right\rangle$ distributions for selected multiplicity intervals.

\section{Systematic uncertainties}

The following sources of systematic uncertainty on the $\mathrm{J} / \psi$ yields in multiplicity classes are considered:

(i) the signal extraction,

(ii) the normalisation,

(iii) the effect of resolution and pile-up,

(iv) the event-by-event $N_{\text {tracklet }}$ to $N_{\text {tracklet }}^{\text {corr }}$ correction, and

(v) the event selection efficiency of the NSD event class.

For the measurement of the yields in each multiplicity interval normalized to the event average, the systematic uncertainties are estimated directly for this ratio. Details on the signal extraction uncertainty were addressed in the previous section. The values are estimated by varying the signal and background shapes of the fit function, as well as by varying the invariant-mass range of the fit. The systematic uncertainty is computed as the root-meansquare of the uncertainties on the ratio for each of these fits, ranging between $0.8-2.3 \%$ $(0.5-1.9 \%)$ at forward (backward) rapidity, being larger at large multiplicities where the 
number of events is smaller. The normalisation factor of the dimuon triggered to $\mathrm{MB}$ events $F_{\text {norm }}$ is studied using three alternative methods [13]. The first method evaluates the probability of a coincidence of a dimuon- and a MB-triggered event in a MB-triggered data set. The second method exploits the higher probability of occurrence of a single-muon trigger by looking at the product of the probability of coincidence of a single-muon- and MB-triggered event and of the probability of finding a dimuon event in the single-muon triggered data. The third method is based on information from the trigger scalers. The run-by-run spread of the $F_{\text {norm }} / F_{\text {norm }}^{i}$ values, ratio of the normalisation values in the integrated and specific multiplicity intervals, computed for these three methods determines a $2.5 \%$ systematic uncertainty, independent of multiplicity. The effect of the method of choice for the event-by-event correction from $N_{\text {tracklet }}$ to $N_{\text {tracklet }}^{\text {corr }}$ on the $\mathrm{J} / \psi$ yield is also studied $[29,30]$. Both the randomisation function (Poisson or binomial) and the reference normalisation of the correction are varied. The Poissonian smearing is applied when the maximum is selected as normalisation reference, while the binomial correction should be used when considering all other possible reference values (in our case the minimum). The influence of these modifications on the yield ranges from $0.1 \%$ to $2.6 \%(4.3 \%)$ at forward (backward) rapidity, as a function of multiplicity.

The uncertainty coming from pile up and multiplicity axis resolution is estimated as a single contribution by repeating the analysis multiple times with a different randomisation seed for the event-by-event correction, or introducing a small shift of the $N_{\text {tracklet }}^{\text {corr }}$ intervals, or varying the pile-up rejection criteria. The uncertainty amounts to $2 \%$, independent of multiplicity. The uncertainty on the event selection efficiency for the NSD event class is estimated as in ref. [48]. The uncertainty amounts to $1 \%$ and is correlated in all multiplicity intervals. Table 2 summarizes all contributions to the systematic uncertainty on the normalized yield.

For the $\left\langle p_{\mathrm{T}}\right\rangle$, the effects of the uncertainty on the $\left\langle p_{\mathrm{T}}\right\rangle$ extraction procedure and of the $A \varepsilon$ are considered. Similar to the yields, the signal extraction uncertainty is estimated by varying the fit function and its range. In addition, as the $S /(S+B)$ terms in eq. 4.2 are fixed in the fit to the $\left\langle p_{\mathrm{T}}\right\rangle$ invariant-mass spectrum, the influence of the statistical uncertainty on the $\mathrm{J} / \psi$ signal $S$ is introduced via a Gaussian smearing of $S$ (with respect to its statistical uncertainty) to prevent artificially minimising the uncertainty. It ranges from $0.2 \%$ to $3.0 \%(1.2 \%)$ at forward (backward) rapidity, increasing with multiplicity as a consequence of the smaller number of events. The uncertainty on the absolute $\left\langle p_{\mathrm{T}}\right\rangle$ also takes into account the uncertainty on:

(i) the $\mathrm{MC}$ input shapes as a function of $p_{\mathrm{T}}$ and $y_{\mathrm{cms}}$, ranging from $<0.1$ to $6 \%(<0.1$ to $11 \%$ ) at forward (backward) rapidity,

(ii) the tracking efficiency, $1 \%$ [13],

(iii) the trigger efficiency, $2.6 \%(3.1 \%)[13]$, and

(iv) the matching efficiency between the tracks in the $\mathrm{MCH}$ and the MTR, 1\% [13].

To evaluate the uncertainty on the $\mathrm{MC}$ input, the data are divided into two multiplicity classes at the mean of the $N_{\text {tracklet }}^{\text {corr }}$ distribution for each rapidity interval. For each of 


\begin{tabular}{|c|c|c|}
\hline Source & $2.03<y_{\mathrm{cms}}<3.53$ & $-4.46<y_{\mathrm{cms}}<-2.96$ \\
\hline Signal extraction & $0.8-2.3 \%$ & $0.5-1.9 \%$ \\
Normalization $\left(F_{\text {norm }}\right)$ & $2.5 \%$ & $2.5 \%$ \\
Event-by-event $N_{\text {tracklet }}^{\text {corr }}$ & $0.1-2.6 \%$ & $0.1-4.3 \%$ \\
Bin-flow and pile-up & $2 \%$ & $2 \%$ \\
Normalization to NSD & $1 \%^{*}$ & $1 \%^{*}$ \\
\hline
\end{tabular}

Table 2. Sources of systematic uncertainties on the normalized yield. The contributions marked with an asterisk are correlated in multiplicity.

\begin{tabular}{|c|cc|cc|}
\hline & \multicolumn{2}{|c|}{$2.03<y_{\mathrm{cms}}<3.53$} & \multicolumn{2}{c|}{$-4.46<y_{\mathrm{cms}}<-2.96$} \\
Source & $\left\langle p_{\mathrm{T}}\right\rangle$ & $\left\langle p_{\mathrm{T}}\right\rangle /\left\langle p_{\mathrm{T}}^{\text {int }}\right\rangle$ & $\left\langle p_{\mathrm{T}}\right\rangle$ & $\left\langle p_{\mathrm{T}}\right\rangle /\left\langle p_{\mathrm{T}}^{\text {int }}\right\rangle$ \\
\hline Signal extraction & $0.2-3.0 \%(0.2 \%)$ & $0.3-3.0 \%$ & $0.2-1.2 \%(0.2 \%)$ & $0.3-1.3 \%$ \\
Tracking efficiency & $1 \%^{*}$ & - & $1 \%^{*}$ & - \\
Trigger efficiency & $2.6 \%^{*}$ & - & $3.1 \%^{*}$ & - \\
Track-trigger matching & $1 \%^{*}$ & - & $1 \%^{*}$ & - \\
Monte Carlo input & $\left\langle 0.1-6 \%^{\diamond}\right.$ & $<0.1-2 \%^{\diamond}$ & $<0.1-11 \%^{\diamond}$ & $<0.1-4 \%^{\diamond}$ \\
\hline
\end{tabular}

Table 3. Systematic uncertainty sources on the average and normalized average $p_{\mathrm{T}}$. The values in parentheses correspond to the multiplicity-integrated uncertainties related to the signal extraction. The contributions marked with an asterisk are correlated in multiplicity. The uncertainty on MC input, marked with a diamond, is partially correlated in multiplicity.

these bins, the $\left\langle p_{\mathrm{T}}\right\rangle$ is estimated using a modified $A \varepsilon$ correction, which was re-weighted to better describe the $p_{\mathrm{T}^{-}}$and $y$-dependent distributions of $\mathrm{J} / \psi$ in given bin. The systematic uncertainty is taken as the difference of the original $\left\langle p_{\mathrm{T}}\right\rangle$ value, computed with the initial $A \varepsilon$ correction, and the new $\left\langle p_{\mathrm{T}}\right\rangle$ estimated with re-weighted correction. The uncertainty on all the measured multiplicity intervals is extrapolated from these two values assuming that in each class the uncertainty is proportional to the $\left\langle p_{\mathrm{T}}\right\rangle$. The contributions of the tracking, the trigger and their matching to the uncertainty are correlated between multiplicity intervals. The normalized $\left\langle p_{\mathrm{T}}\right\rangle$ values are only affected by the uncertainty on the signal extraction procedure and the MC input, which is partly correlated in multiplicity and ranges from $<0.1 \%$ to $2 \%(<0.1 \%$ to $4 \%)$ in the forward (backward) rapidity interval. Table 3 summarizes all contributions to the average, $\left\langle p_{\mathrm{T}}\right\rangle$, and normalized average, $\left\langle p_{\mathrm{T}}\right\rangle /\left\langle p_{\mathrm{T}}^{\mathrm{int}}\right\rangle, p_{\mathrm{T}}$ measurements. The correlated uncertainties are added in quadrature and quoted in the plot as a text.

\section{Results and discussion}

The normalized $\mathrm{J} / \psi$ yield, at forward and backward rapidities, is presented in figure 3 as a function of the normalized charged-particle pseudorapidity density, measured at midrapidity $(|\eta|<1)$. The normalized yield increases with increasing multiplicity in both rapidity intervals. The yield at backward rapidity grows faster than the one at forward rapidity, reaching values above those expected from a linear (with slope unity) increase at large 


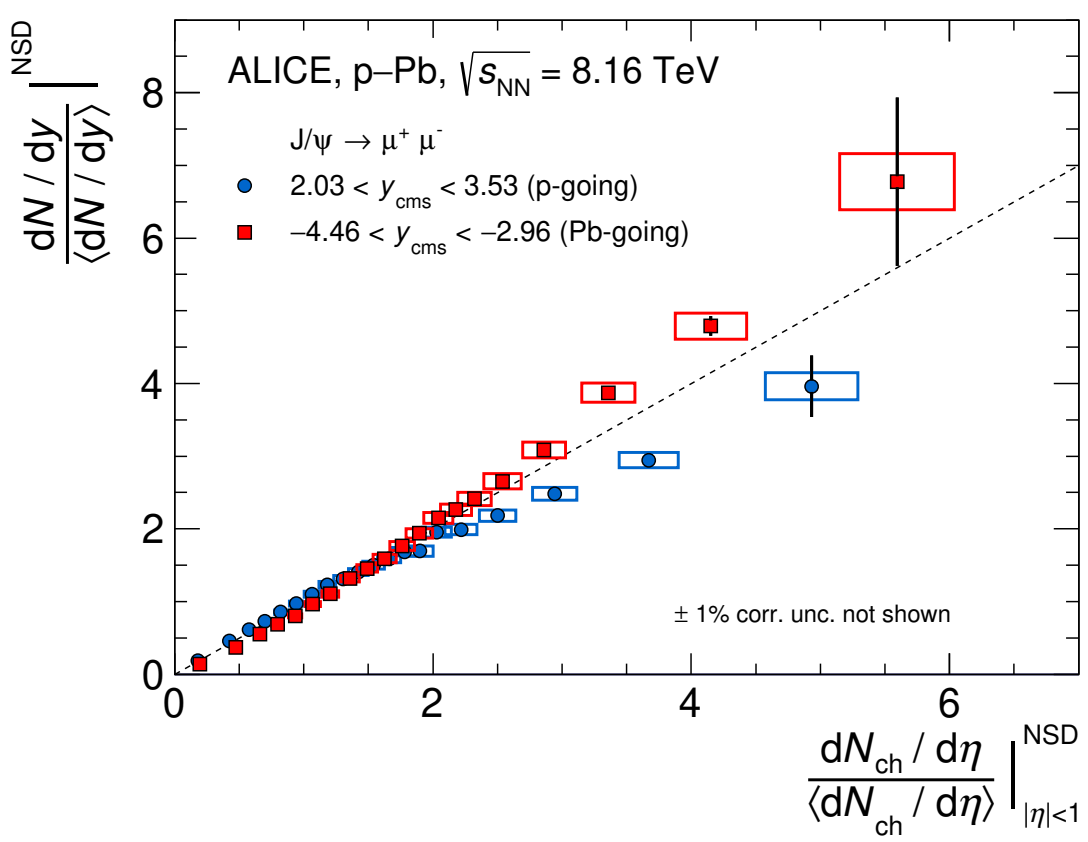

Figure 3. Normalized yield of inclusive $\mathrm{J} / \psi$, at forward and backward rapidities, as a function of the normalized charged-particle pseudorapidity density, measured at midrapidity, in p-Pb collisions at $\sqrt{s_{\mathrm{NN}}}=8.16 \mathrm{TeV}$. The vertical bars represent the statistical uncertainties. The vertical and horizontal widths of the boxes represent the respective systematic uncertainties for the $\mathrm{J} / \psi$ yields and the multiplicities. The dashed line indicates the one-to-one correlation, to guide the eye.

multiplicities. On the other hand, at forward rapidity the values show a slower-than-linear increase at large multiplicities. The forward and backward rapidity yields cross a linear increase estimate (and each other) at around 1.5 times the average multiplicity. The underlying mechanism remains unclear. The forward (p-going) rapidity region probes the Pb-nucleus low Bjorken- $x$ regime $\left(x_{\mathrm{Pb}} \sim 10^{-5}\right.$ in a naive 2-body calculation for $\left.p_{\mathrm{T}}=0\right)$, whereas the backward (Pb-going) rapidity is sensitive to the intermediate-to-large values $\left(x_{\mathrm{Pb}} \sim 10^{-2}\right)$. The observed suppression of the $p_{\mathrm{T}^{-}}$and multiplicity-integrated $\mathrm{J} / \psi$ yield at forward rapidity, with respect to pp collisions, is described by different cold nuclear matter models considering the probed shadowing/saturation domain [13]. The centralitydifferential measurements at $\sqrt{s_{\mathrm{NN}}}=5.02 \mathrm{TeV}[23]$ of the nuclear modification factor, $\left\langle p_{\mathrm{T}}\right\rangle$ and $\left\langle p_{\mathrm{T}}^{2}\right\rangle$, corresponding to relative multiplicities of at most 2.5 times the average one, can also be described by these models. The contribution from beauty-quark decays to the inclusive $\mathrm{J} / \psi$ yield amounts to $\sim 10 \%$ [57]. It is not expected to affect significantly these results, since a similar trend was observed for prompt and non-prompt $\mathrm{J} / \psi$ as a function of the charged-particle pseudorapidity density in pp collisions [30]. Moreover, the autocorrelations influence is negligible in this analysis due to the large rapidity gap between the measurement of the charged-particle multiplicity and the $\mathrm{J} / \psi$ yield [58].

Figure 4 presents $\left\langle p_{\mathrm{T}}\right\rangle$ as a function of the relative charged-particle pseudorapidity density, in p-Pb collisions at $\sqrt{s_{\mathrm{NN}}}=8.16 \mathrm{TeV}$. The measured $\left\langle p_{\mathrm{T}}\right\rangle$ is systematically smaller 


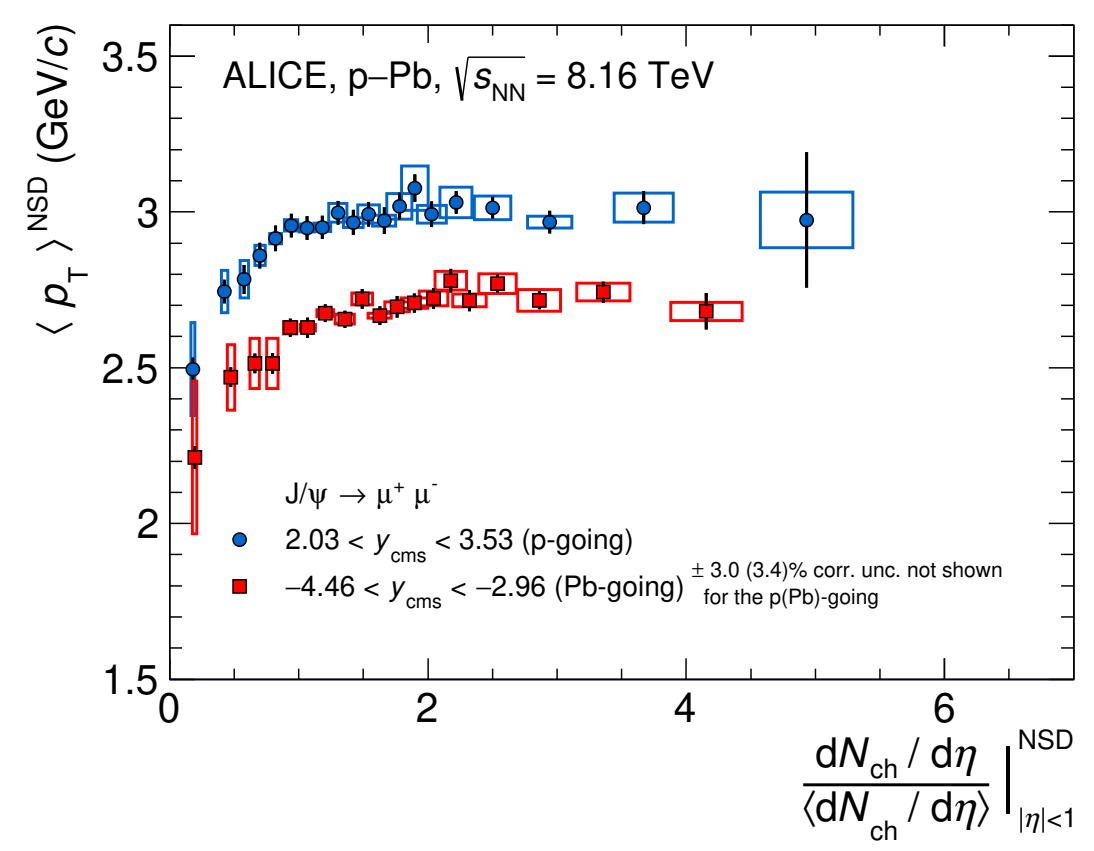

Figure 4. Average transverse momentum of inclusive $\mathrm{J} / \psi$ at forward and backward rapidities as a function of the normalized charged-particle pseudorapidity density, measured at midrapidity, in $\mathrm{p}-\mathrm{Pb}$ collisions at $\sqrt{s_{\mathrm{NN}}}=8.16 \mathrm{TeV}$. The vertical bars represent the statistical uncertainties, the boxes the systematic ones.

at backward than at forward rapidity. This is also true for the multiplicity-integrated value, which is consistent with the observed decrease of $\left\langle p_{\mathrm{T}}\right\rangle$ with increasing $\left|y_{\mathrm{cms}}\right|$ in pp collisions [59]. The $\left\langle p_{\mathrm{T}}\right\rangle$ increases steadily for multiplicities below the average, and saturates above the average multiplicity. Two naive scenarios are typically considered to explain high-multiplicity events: the incoherent superposition of multiple parton-parton collisions, or single parton interactions with higher energy transfer. One would expect the latter to be characterized by a higher $\left\langle p_{\mathrm{T}}\right\rangle$ of the produced $\mathrm{J} / \psi$. Reality is probably somewhere in between these two simplified scenarios. The simultaneous increase of the $\mathrm{p}-\mathrm{Pb}$ yield together with the saturation of $\left\langle p_{\mathrm{T}}\right\rangle$ at large multiplicities may point to $\mathrm{J} / \psi$ production from an incoherent superposition of parton-parton collisions.

The measured yield in $\mathrm{p}-\mathrm{Pb}$ collisions can be described with the EPOS 3 event generator $[60,61]$ (see figure 5) based on a combination of Gribov-Regge theory and pQCD: where the individual scatterings are identified with parton ladders emerging as flux tubes, the existence of multiple nucleon-nucleon collisions in $\mathrm{p}-\mathrm{Pb}$ collisions is accounted for, the initial conditions of the collision are modified due to CNM effects including parton saturation, and slow string segments (far from the surface) can be further mapped to fluid dynamic fields using a core-corona description. The $\mathrm{J} / \psi$ bound-state formation in EPOS 3 assumes a color-evaporation approach, i.e. it is associated to a charm quark-anti-quark pair in a given mass range. The influence of the $3 \mathrm{D}+1$ viscous hydrodynamic evolution of the bulk (starting from flux tube initial conditions) in the EPOS 3 calculation is small (see figure 5). 

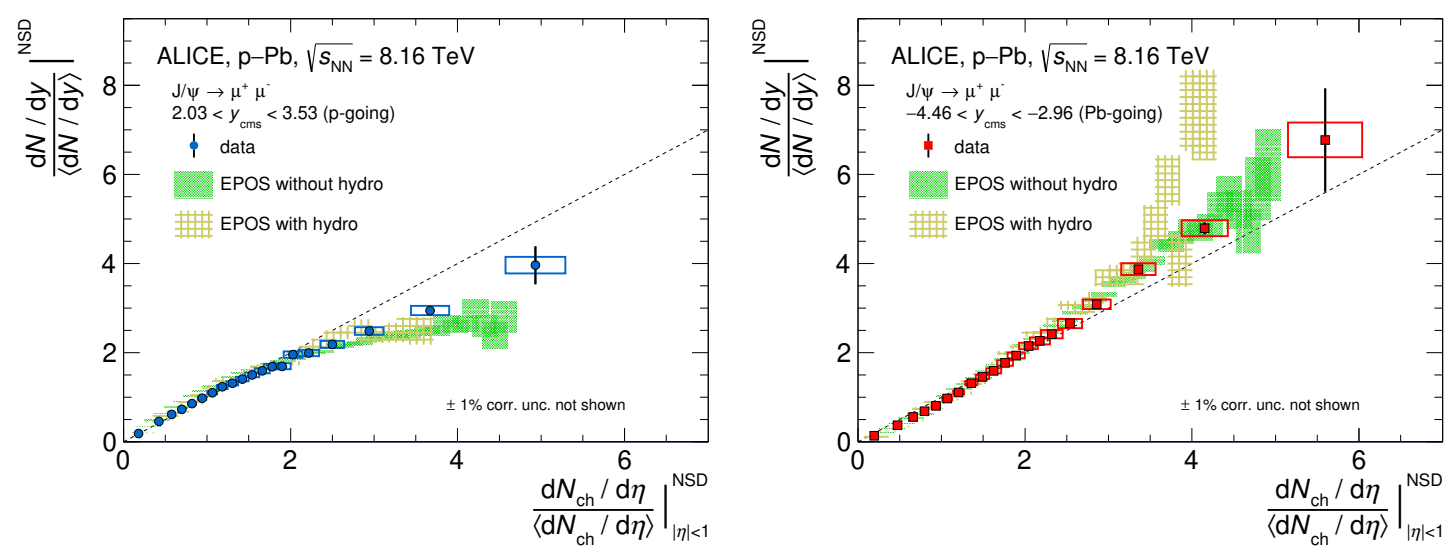

Figure 5. Normalized yield of inclusive $\mathrm{J} / \psi$ as a function of the normalized charged-particle pseudorapidity density, measured at midrapidity, in p- $\mathrm{Pb}$ collisions at $\sqrt{s_{\mathrm{NN}}}=8.16 \mathrm{TeV}$ compared with EPOS $3[60,61]$ calculations. Left (right) panel presents the measurement at forward (backward) rapidity. The vertical bars represent the statistical uncertainties, the boxes the systematic ones. The dashed line indicates the one-to-one correlation, to guide the eye. The shaded areas represent the statistical uncertainties on the EPOS 3 calculations.

However the number of simulated events at large multiplicities is limited and does not allow us to elucidate possible hydrodynamic effects. EPOS 3 description of the measurement suggests $\mathrm{J} / \psi$ production from an incoherent superposition of parton-parton collisions.

The normalized $\mathrm{J} / \psi$ yield and $\left\langle p_{\mathrm{T}}\right\rangle$ are compared with the results in $\mathrm{p}-\mathrm{Pb}$ collisions at $\sqrt{s_{\mathrm{NN}}}=5.02 \mathrm{TeV}$ [26] in figure 6 and 7, respectively. The measurements are in remarkable agreement, within the uncertainties, at both energies and rapidities. These results extend the probed charged-particle pseudorapidity density interval, both at low and high multiplicity, examining events of up to almost six times the average value. The more precise $\sqrt{s_{\mathrm{NN}}}=8.16 \mathrm{TeV}$ data evidence a continuous increase of the normalized yield with multiplicity up to the largest multiplicities attained. The similarities at $\sqrt{s_{\mathrm{NN}}}=8.16 \mathrm{TeV}$ and $\sqrt{s_{\mathrm{NN}}}=5.02 \mathrm{TeV}$ suggest a common origin of the multiplicity trend, with a mechanism whose effect varies with rapidity, but might have a small dependence on the collision energy, in the explored interval. This is consistent with the large variation of the probed $x_{\mathrm{Pb}}$ with rapidity and its relative slow evolution on the collision energy (typically a factor of 2 in the simplified 2-body picture).

Figure 8 presents a comparison of the normalized $\mathrm{J} / \psi \mathrm{p}-\mathrm{Pb}$ yields with results from pp collisions at $\sqrt{s_{\mathrm{NN}}}=7 \mathrm{TeV}[29]\left(2.5<y_{\mathrm{cms}}<4.0\right)$ and $\mathrm{Pb}-\mathrm{Pb}$ collisions at $\sqrt{s_{\mathrm{NN}}}=$ $5.02 \mathrm{TeV}$ [62] $\left(2.5<y_{\mathrm{cms}}<4.0\right)$. The corresponding $\left\langle\mathrm{d} N_{\mathrm{ch}} / \mathrm{d} \eta\right\rangle$ in $|\eta|<1$ for those measurements is $6.01 \pm 0.01$ (stat.) ${ }_{-0.12}^{+0.20}$ (syst.) [29] and $544.7 \pm 0.2$ (stat.) \pm 7.3 (syst.) for 0-90\% centrality [63], respectively. The ratio of the yields over the corresponding chargedparticle multiplicity is also shown in figure 8 . The trend exhibited by the pp data is similar to the one observed in the backward (Pb-going) direction. It should be noted that the pp results are normalized to the inelastic 'INEL' event class, whereas the $\mathrm{p}-\mathrm{Pb}$ measurements are normalized to the non-single-diffractive 'NSD' one. In p-Pb collisions these two event classes mostly overlap when comparing $\mathrm{MB}$ results [48]. The $\mathrm{Pb}-\mathrm{Pb}$ data also show a faster- 

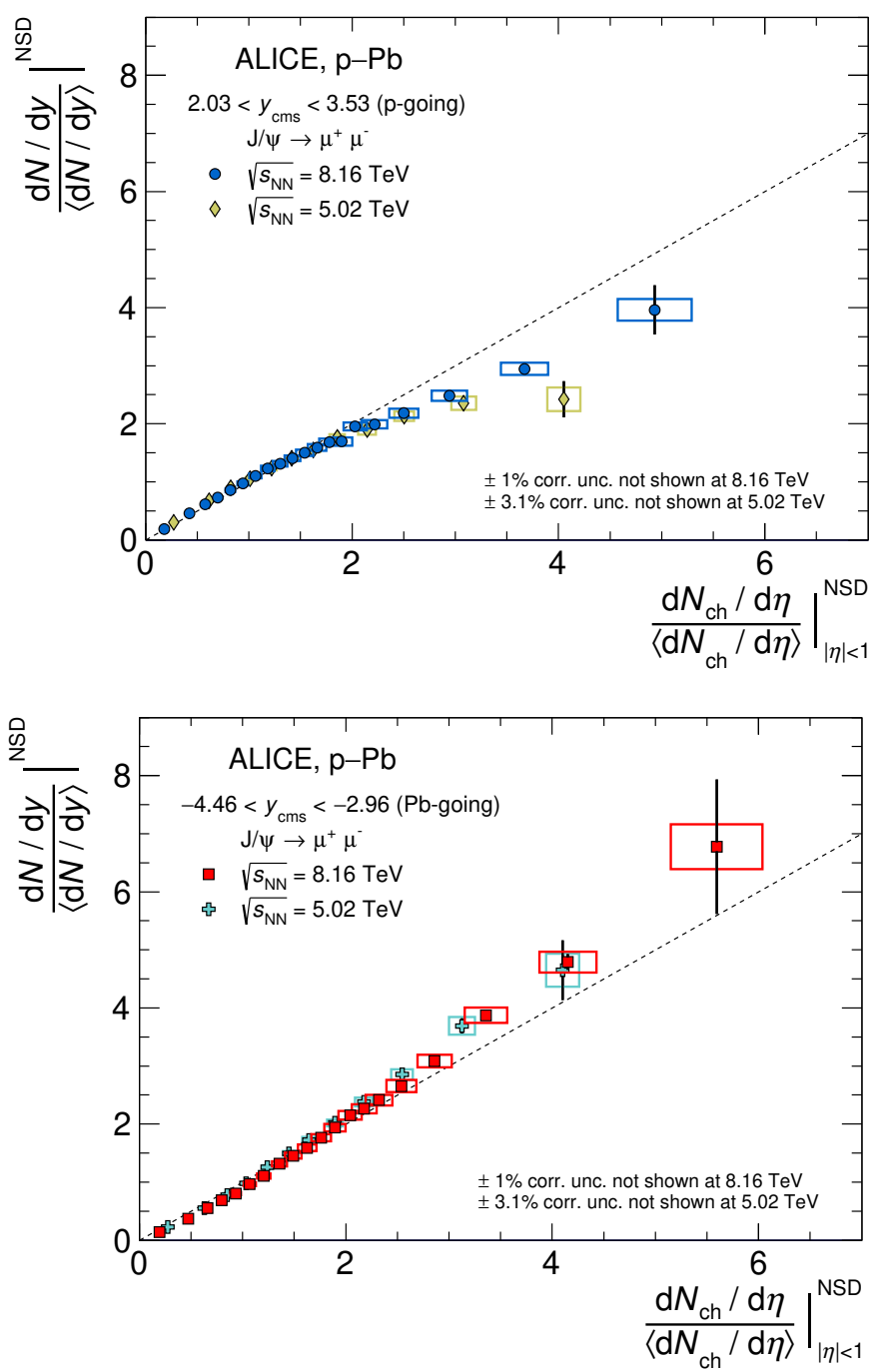

Figure 6. Normalized yield of inclusive $\mathrm{J} / \psi$ as a function of the normalized charged-particle pseudorapidity density, measured at midrapidity, in p- $\mathrm{Pb}$ collisions at $\sqrt{s_{\mathrm{NN}}}=8.16 \mathrm{TeV}$ and $\sqrt{s_{\mathrm{NN}}}=5.02 \mathrm{TeV}[26]$. The top (bottom) panel presents the measurement at forward (backward) rapidity. The vertical bars represent the statistical uncertainties, the boxes the systematic ones. The dashed line indicates the one-to-one correlation, to guide the eye.

than-linear increase with the normalized charged-particle pseudorapidity density. They are compatible within uncertainties with the $\mathrm{p}-\mathrm{Pb}$ backward rapidity result in the restricted multiplicity interval of the measurement. Whereas the pp and $\mathrm{p}-\mathrm{Pb}$ data include $\mathrm{J} / \psi$ with $p_{\mathrm{T}}>0$, the $\mathrm{Pb}-\mathrm{Pb}$ data points include $\mathrm{J} / \psi$ with $0.3<p_{\mathrm{T}}<12 \mathrm{GeV} / c$ to reduce the low- $p_{\mathrm{T}}$ contribution from photoproduction, which is significant only in more peripheral collisions $[62,64,65]$. The overall increase of multiple parton-parton collisions with the colliding system (from pp up to $\mathrm{Pb}-\mathrm{Pb}$ collisions) described in [66] is expected to cancel in the relative quantities reported in this publication, sensitive to the relative evolution with charged-particle density in a given colliding system. Model calculations are needed to 

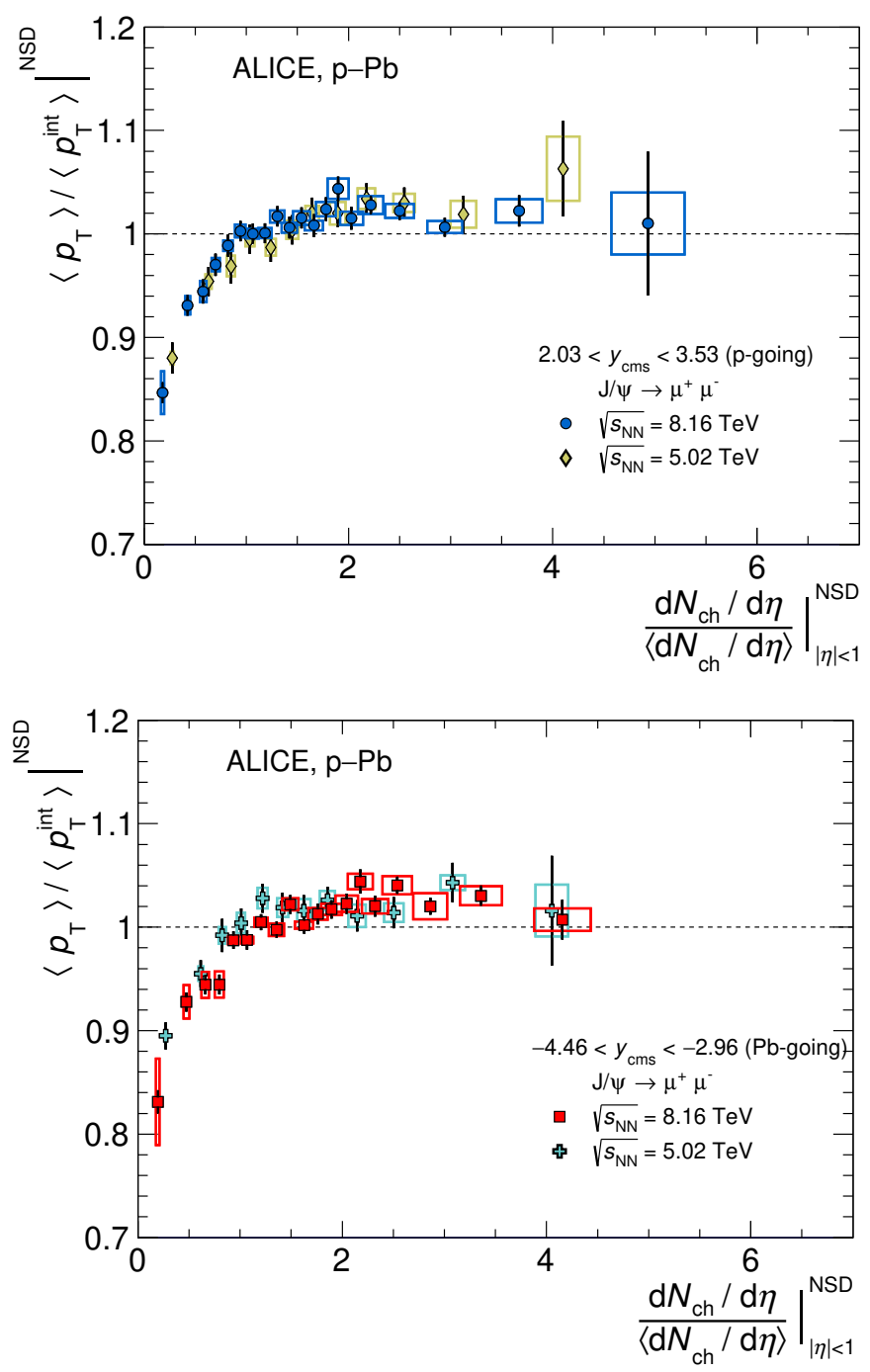

Figure 7. Normalized average transverse momentum of inclusive $\mathrm{J} / \psi$ as a function of the normalized charged-particle pseudorapidity density, measured at midrapidity, in $\mathrm{p}-\mathrm{Pb}$ collisions at $\sqrt{s_{\mathrm{NN}}}=8.16 \mathrm{TeV}$ and $\sqrt{s_{\mathrm{NN}}}=5.02 \mathrm{TeV}[26]$. Top (bottom) panel presents the measurement at forward (backward) rapidity. The vertical bars represent the statistical uncertainties, the boxes the systematic ones.

interpret the similarities of $\mathrm{pp}, \mathrm{p}-\mathrm{Pb}$, and $\mathrm{Pb}-\mathrm{Pb}$ normalized $\mathrm{J} / \psi$ yields at large rapidity as a function of the normalized charged-particle pseudorapidity density at midrapidity.

\section{Conclusions}

The production of inclusive $\mathrm{J} / \psi$ at large rapidities in $\mathrm{p}-\mathrm{Pb}$ collisions at $\sqrt{s_{\mathrm{NN}}}=8.16 \mathrm{TeV}$ is reported as a function of the charged-particle pseudorapidity density at midrapidity. The normalized $\mathrm{J} / \psi$ yield shows an increase with increasing normalised charged-particle pseudorapidity density. The yield at backward rapidity grows faster than the forward rapidity one, reaching values above those of the linear (with slope unity) increase estimate at large 

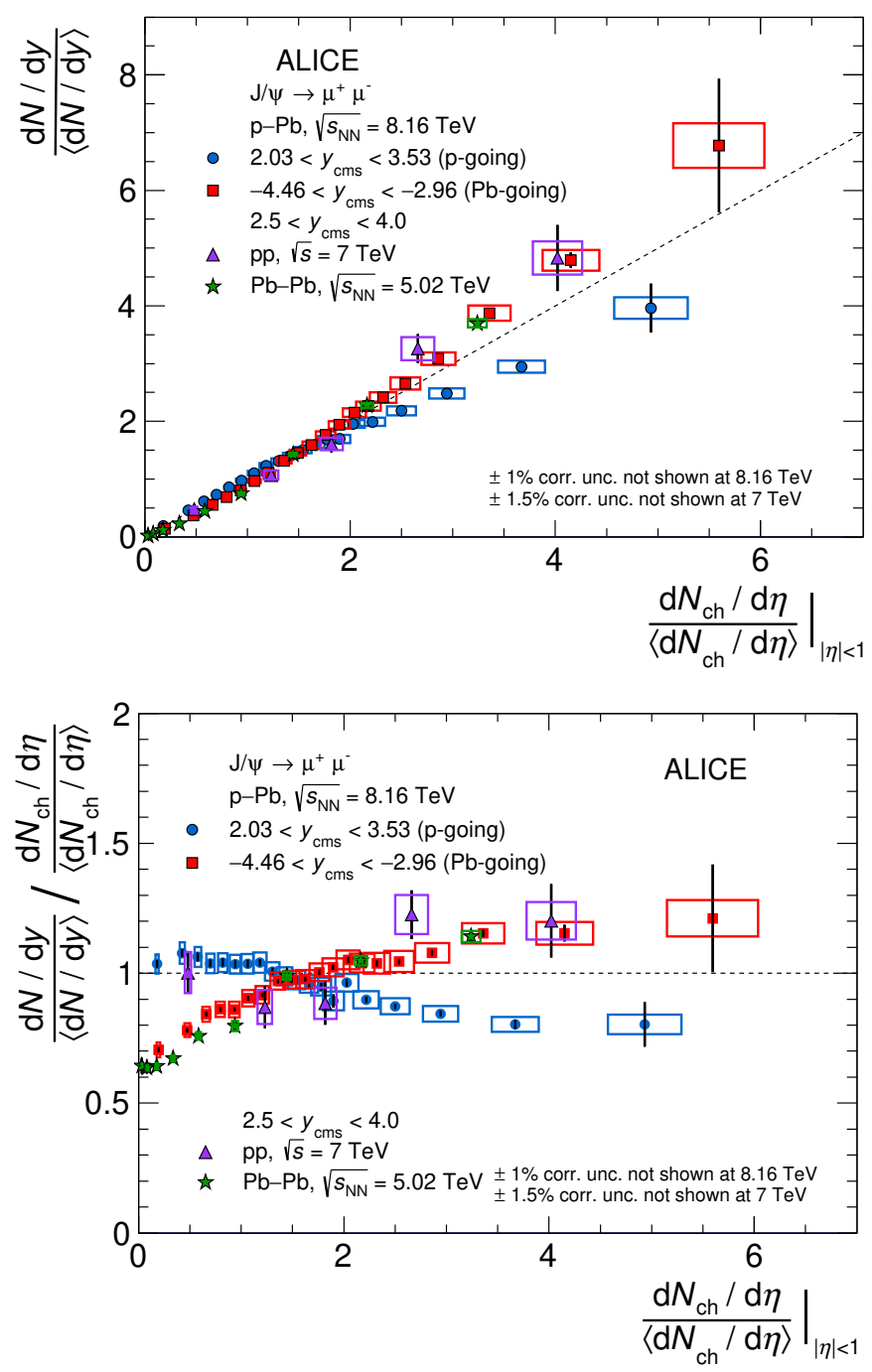

Figure 8. Top: normalized yield of inclusive $\mathrm{J} / \psi$ as a function of the normalized charged-particle pseudorapidity density, measured at midrapidity, in various collision systems. Bottom: ratio of the normalized yields to the corresponding normalized charged-particle pseudorapidity density. The pp results are normalized to INEL collisions [29], whereas p-Pb ones refer to the NSD event class; all for $p_{\mathrm{T}}>0$. The $\mathrm{Pb}-\mathrm{Pb}$ data points include $\mathrm{J} / \psi$ with $0.3<p_{\mathrm{T}}<12 \mathrm{GeV} / c$ to reduce the low- $p_{\mathrm{T}}$ contribution from photoproduction, which is significant only in more peripheral collisions $[62,64$, 65]. The vertical bars represent the statistical uncertainties, the boxes the systematic ones. The dashed line indicates the one-to-one correlation, to guide the eye.

normalised multiplicity, whereas the values at forward rapidity show a slower-than-linear increase. The trends of the normalised yield are reproduced by the EPOS $3[60,61]$ event generator. The $\left\langle p_{\mathrm{T}}\right\rangle$ is smaller at backward than at forward rapidity, consistent with the expected softening of the spectra with increasing $\left|y_{\mathrm{cms}}\right|$. The $\left\langle p_{\mathrm{T}}\right\rangle$ increases steadily for multiplicities below the average, and saturates above the average multiplicity. The simultaneous increase of the yield together with the saturation of $\left\langle p_{\mathrm{T}}\right\rangle$ may point to $\mathrm{J} / \psi$ production from an incoherent superposition of parton-parton collisions. These measurements show 
trends compatible with those observed at $\sqrt{s_{\mathrm{NN}}}=5.02 \mathrm{TeV}[26]$ in p-Pb collisions, but in this work an improved precision and extended multiplicity coverage were reached. The similarities suggest a common origin, with a mechanism whose effect varies with rapidity, but with only a small dependence (if any) on the collision energy.

\section{Acknowledgments}

The ALICE Collaboration would like to thank all its engineers and technicians for their invaluable contributions to the construction of the experiment and the CERN accelerator teams for the outstanding performance of the LHC complex. The ALICE Collaboration gratefully acknowledges the resources and support provided by all Grid centres and the Worldwide LHC Computing Grid (WLCG) collaboration. The ALICE Collaboration acknowledges the following funding agencies for their support in building and running the ALICE detector: A. I. Alikhanyan National Science Laboratory (Yerevan Physics Institute) Foundation (ANSL), State Committee of Science and World Federation of Scientists (WFS), Armenia; Austrian Academy of Sciences, Austrian Science Fund (FWF): [M 2467N36] and Nationalstiftung für Forschung, Technologie und Entwicklung, Austria; Ministry of Communications and High Technologies, National Nuclear Research Center, Azerbaijan; Conselho Nacional de Desenvolvimento Científico e Tecnológico (CNPq), Financiadora de Estudos e Projetos (Finep), Fundação de Amparo à Pesquisa do Estado de São Paulo (FAPESP) and Universidade Federal do Rio Grande do Sul (UFRGS), Brazil; Ministry of Education of China (MOEC), Ministry of Science \& Technology of China (MSTC) and National Natural Science Foundation of China (NSFC), China; Ministry of Science and Education and Croatian Science Foundation, Croatia; Centro de Aplicaciones Tecnológicas y Desarrollo Nuclear (CEADEN), Cubaenergía, Cuba; Ministry of Education, Youth and Sports of the Czech Republic, Czech Republic; The Danish Council for Independent Research - Natural Sciences, the VILLUM FONDEN and Danish National Research Foundation (DNRF), Denmark; Helsinki Institute of Physics (HIP), Finland; Commissariat à l'Energie Atomique (CEA) and Institut National de Physique Nucléaire et de Physique des Particules (IN2P3) and Centre National de la Recherche Scientifique (CNRS), France; Bundesministerium für Bildung und Forschung (BMBF) and GSI Helmholtzzentrum für Schwerionenforschung GmbH, Germany; General Secretariat for Research and Technology, Ministry of Education, Research and Religions, Greece; National Research, Development and Innovation Office, Hungary; Department of Atomic Energy Government of India (DAE), Department of Science and Technology, Government of India (DST), University Grants Commission, Government of India (UGC) and Council of Scientific and Industrial Research (CSIR), India; Indonesian Institute of Science, Indonesia; Centro Fermi - Museo Storico della Fisica e Centro Studi e Ricerche Enrico Fermi and Istituto Nazionale di Fisica Nucleare (INFN), Italy; Institute for Innovative Science and Technology, Nagasaki Institute of Applied Science (IIST), Japanese Ministry of Education, Culture, Sports, Science and Technology (MEXT) and Japan Society for the Promotion of Science (JSPS) KAKENHI, Japan; Consejo Nacional de Ciencia (CONACYT) y Tecnología, through Fondo de Cooperación Internacional en Ciencia y Tecnología (FONCICYT) and Dirección Gen- 
eral de Asuntos del Personal Academico (DGAPA), Mexico; Nederlandse Organisatie voor Wetenschappelijk Onderzoek (NWO), Netherlands; The Research Council of Norway, Norway; Commission on Science and Technology for Sustainable Development in the South (COMSATS), Pakistan; Pontificia Universidad Católica del Perú, Peru; Ministry of Science and Higher Education, National Science Centre and WUT ID-UB, Poland; Korea Institute of Science and Technology Information and National Research Foundation of Korea (NRF), Republic of Korea; Ministry of Education and Scientific Research, Institute of Atomic Physics and Ministry of Research and Innovation and Institute of Atomic Physics, Romania; Joint Institute for Nuclear Research (JINR), Ministry of Education and Science of the Russian Federation, National Research Centre Kurchatov Institute, Russian Science Foundation and Russian Foundation for Basic Research, Russia; Ministry of Education, Science, Research and Sport of the Slovak Republic, Slovakia; National Research Foundation of South Africa, South Africa; Swedish Research Council (VR) and Knut \& Alice Wallenberg Foundation (KAW), Sweden; European Organization for Nuclear Research, Switzerland; Suranaree University of Technology (SUT), National Science and Technology Development Agency (NSDTA) and Office of the Higher Education Commission under NRU project of Thailand, Thailand; Turkish Atomic Energy Agency (TAEK), Turkey; National Academy of Sciences of Ukraine, Ukraine; Science and Technology Facilities Council (STFC), United Kingdom; National Science Foundation of the United States of America (NSF) and United States Department of Energy, Office of Nuclear Physics (DOE NP), United States of America.

Open Access. This article is distributed under the terms of the Creative Commons Attribution License (CC-BY 4.0), which permits any use, distribution and reproduction in any medium, provided the original author(s) and source are credited.

\section{References}

[1] P. Braun-Munzinger and J. Stachel, The quest for the quark-gluon plasma, Nature 448 (2007) 302 [INSPIRE].

[2] T. Matsui and H. Satz, $J / \psi$ suppression by quark-gluon plasma formation, Phys. Lett. B 178 (1986) 416 [INSPIRE].

[3] NA50 collaboration, A new measurement of $\mathrm{J} / \psi$ suppression in $\mathrm{Pb}-\mathrm{Pb}$ collisions at $158 \mathrm{GeV}$ per nucleon, Eur. Phys. J. C 39 (2005) 335 [hep-ex/0412036] [INSPIRE].

[4] NA60 collaboration, $J / \psi$ production in indium-indium collisions at $158 \mathrm{GeV} /$ nucleon, Phys. Rev. Lett. 99 (2007) 132302 [INSPIRE].

[5] PHENIX collaboration, $J / \psi$ suppression at forward rapidity in Au+Au collisions at $\sqrt{s_{\mathrm{NN}}}=200 \mathrm{GeV}$, Phys. Rev. C 84 (2011) 054912 [arXiv:1103.6269] [INSPIRE].

[6] ALICE collaboration, $J / \psi$ suppression at forward rapidity in $\mathrm{Pb}-\mathrm{Pb}$ collisions at $\sqrt{s_{\mathrm{NN}}}=2.76 \mathrm{TeV}$, Phys. Rev. Lett. 109 (2012) 072301 [arXiv:1202.1383] [INSPIRE].

[7] ALICE collaboration, Centrality, rapidity and transverse momentum dependence of $\mathrm{J} / \psi$ suppression in $\mathrm{Pb}-\mathrm{Pb}$ collisions at $\sqrt{s_{\mathrm{NN}}}=2.76 \mathrm{TeV}$, Phys. Lett. B 734 (2014) 314 [arXiv: 1311.0214] [INSPIRE]. 
[8] STAR collaboration, $J / \psi$ production at low $p_{T}$ in $A u+A u$ and $C u+C u$ collisions at $\sqrt{s_{\mathrm{NN}}}=200 \mathrm{GeV}$ with the STAR detector, Phys. Rev. C 90 (2014) 024906 [arXiv: 1310.3563] [INSPIRE].

[9] CMS collaboration, Suppression of non-prompt $J / \psi$, prompt $J / \psi$, and $Y(1 S)$ in $P b P b$ collisions at $\sqrt{s_{\mathrm{NN}}}=2.76 \mathrm{TeV}$, JHEP 05 (2012) 063 [arXiv:1201.5069] [INSPIRE].

[10] ATLAS collaboration, Measurement of the centrality dependence of $J / \psi$ yields and observation of $Z$ production in lead-lead collisions with the ATLAS detector at the LHC, Phys. Lett. B 697 (2011) 294 [arXiv:1012.5419] [InSPIRE].

[11] ALICE collaboration, $J / \psi$ suppression at forward rapidity in $\mathrm{Pb}$ - $\mathrm{Pb}$ collisions at $\sqrt{s_{\mathrm{NN}}}=5.02 \mathrm{TeV}$, Phys. Lett. B 766 (2017) 212 [arXiv:1606.08197] [INSPIRE].

[12] ALICE collaboration, $J / \psi$ production and nuclear effects in $p$-Pb collisions at $\sqrt{s_{\mathrm{NN}}}=5.02$ TeV, JHEP 02 (2014) 073 [arXiv: 1308.6726] [inSPIRE].

[13] ALICE collaboration, Inclusive $J / \psi$ production at forward and backward rapidity in $p-P b$ collisions at $\sqrt{s_{\mathrm{NN}}}=8.16 \mathrm{TeV}$, JHEP 07 (2018) 160 [arXiv:1805.04381] [INSPIRE].

[14] LHCb collaboration, Study of $J / \psi$ production and cold nuclear matter effects in $p P b$ collisions at $\sqrt{s_{\mathrm{NN}}}=5 \mathrm{TeV}$, JHEP 02 (2014) 072 [arXiv:1308.6729] [INSPIRE].

[15] J.L. Albacete et al., Predictions for $p+P b$ collisions at $\sqrt{s_{\mathrm{NN}}}=5 \mathrm{TeV}$ : comparison with data, Int. J. Mod. Phys. E 25 (2016) 1630005 [arXiv: 1605.09479] [INSPIRE].

[16] A. Kusina, J.-P. Lansberg, I. Schienbein and H.-S. Shao, Gluon shadowing in heavy-flavor production at the LHC, Phys. Rev. Lett. 121 (2018) 052004 [arXiv:1712.07024] [InSPIRE].

[17] Y.-Q. Ma, R. Venugopalan, K. Watanabe and H.-F. Zhang, $\psi(2 S)$ versus $J / \psi$ suppression in proton-nucleus collisions from factorization violating soft color exchanges, Phys. Rev. C 97 (2018) 014909 [arXiv:1707.07266] [INSPIRE].

[18] B. Ducloué, T. Lappi, and H. Mäntysaari, Forward $J / \psi$ and $D$ meson nuclear suppression at the LHC, Nucl. Part. Phys. Proc. 289-290 (2017) 309 [arXiv: 1612.04585] [InSPIRE].

[19] F. Arleo and S. Peigné, Quarkonium suppression in heavy-ion collisions from coherent energy loss in cold nuclear matter, JHEP 10 (2014) 073 [arXiv:1407.5054] [INSPIRE].

[20] ALICE collaboration, Suppression of $\psi(2 S)$ production in $p$-Pb collisions at $\sqrt{s_{\mathrm{NN}}}=5.02$ TeV, JHEP 12 (2014) 073 [arXiv:1405.3796] [InSPIRE].

[21] E.G. Ferreiro, Excited charmonium suppression in proton-nucleus collisions as a consequence of comovers, Phys. Lett. B $\mathbf{7 4 9}$ (2015) 98 [arXiv:1411.0549] [INSPIRE].

[22] B. Chen, T. Guo, Y. Liu and P. Zhuang, Cold and hot nuclear matter effects on charmonium production in $p+P b$ collisions at LHC energy, Phys. Lett. B 765 (2017) 323 [arXiv: 1607.07927] [INSPIRE].

[23] ALICE collaboration, Centrality dependence of inclusive $J / \psi$ production in $p$ - $P b$ collisions at $\sqrt{s_{\mathrm{NN}}}=5.02 \mathrm{TeV}$, JHEP 11 (2015) 127 [arXiv: 1506.08808] [INSPIRE].

[24] ALICE collaboration, Centrality dependence of $\psi(2 S)$ suppression in $p$-Pb collisions at $\sqrt{s_{\mathrm{NN}}}=5.02 \mathrm{TeV}, J H E P 06(2016) 050$ [arXiv: 1603.02816] [INSPIRE].

[25] ALICE collaboration, Centrality dependence of particle production in $p$-Pb collisions at $\sqrt{s_{\mathrm{NN}}}=5.02 \mathrm{TeV}$, Phys. Rev. C 91 (2015) 064905 [arXiv:1412.6828] [INSPIRE]. 
[26] ALICE collaboration, $J / \psi$ production as a function of charged-particle pseudorapidity density in $p$-Pb collisions at $\sqrt{s_{\mathrm{NN}}}=5.02$ TeV, Phys. Lett. B 776 (2018) 91 [arXiv: 1704.00274] [INSPIRE].

[27] CMS collaboration, Event activity dependence of $Y(n S)$ production in $\sqrt{s_{\mathrm{NN}}}=5.02 \mathrm{TeV}$ $p P b$ and $\sqrt{s}=2.76 \mathrm{TeV} p p$ collisions, JHEP 04 (2014) 103 [arXiv:1312.6300] [INSPIRE].

[28] ATLAS collaboration, Measurement of quarkonium production in proton-lead and proton-proton collisions at $5.02 \mathrm{TeV}$ with the ATLAS detector, Eur. Phys. J. C 78 (2018) 171 [arXiv: 1709. 03089] [INSPIRE].

[29] ALICE collaboration, $J / \psi$ production as a function of charged particle multiplicity in $p p$ collisions at $\sqrt{s}=7 \mathrm{TeV}$, Phys. Lett. B 712 (2012) 165 [arXiv:1202.2816] [INSPIRE].

[30] ALICE collaboration, Measurement of charm and beauty production at central rapidity versus charged-particle multiplicity in proton-proton collisions at $\sqrt{s}=7 \mathrm{TeV}$, JHEP 09 (2015) 148 [arXiv: 1505.00664] [INSPIRE].

[31] ALICE collaboration, Measurement of D-meson production versus multiplicity in $\mathrm{p}$ - $\mathrm{Pb}$ collisions at $\sqrt{s_{\mathrm{NN}}}=5.02 \mathrm{TeV}$, JHEP 08 (2016) 078 [arXiv: 1602.07240] [INSPIRE].

[32] CMS collaboration, Observation of long-range near-side angular correlations in proton-proton collisions at the LHC, JHEP 09 (2010) 091 [arXiv: 1009.4122] [INSPIRE].

[33] CMS collaboration, Observation of long-range near-side angular correlations in proton-lead collisions at the LHC, Phys. Lett. B 718 (2013) 795 [arXiv:1210.5482] [INSPIRE].

[34] ALICE collaboration, Long-range angular correlations on the near and away side in $\mathrm{p}-\mathrm{Pb}$ collisions at $\sqrt{s_{\mathrm{NN}}}=5.02 \mathrm{TeV}$, Phys. Lett. B $\mathbf{7 1 9}$ (2013) 29 [arXiv:1212.2001] [INSPIRE].

[35] ATLAS collaboration, Observation of associated near-side and away-side long-range correlations in $\sqrt{s_{\mathrm{NN}}}=5.02 \mathrm{TeV}$ proton-lead collisions with the ATLAS detector, Phys. Rev. Lett. 110 (2013) 182302 [arXiv: 1212.5198] [INSPIRE].

[36] ALICE collaboration, Harmonic decomposition of two-particle angular correlations in $\mathrm{Pb}-\mathrm{Pb}$ collisions at $\sqrt{s_{\mathrm{NN}}}=2.76 \mathrm{TeV}$, Phys. Lett. B 708 (2012) 249 [arXiv:1109.2501] [InSPIRE].

[37] ALICE collaboration, Search for collectivity with azimuthal $J / \psi$-hadron correlations in high multiplicity $p-P b$ collisions at $\sqrt{s_{\mathrm{NN}}}=5.02$ and 8.16 TeV, Phys. Lett. B $\mathbf{7 8 0}$ (2018) 7 [arXiv: 1709.06807] [INSPIRE].

[38] CMS collaboration, Observation of prompt $J / \psi$ meson elliptic flow in high-multiplicity $p P b$ collisions at $\sqrt{s_{\mathrm{NN}}}=8.16 \mathrm{TeV}$, Phys. Lett. B 791 (2019) 172 [arXiv:1810.01473] [INSPIRE].

[39] ALICE collaboration, $J / \psi$ elliptic flow in Pb-Pb collisions at $\sqrt{s_{\mathrm{NN}}}=5.02 \mathrm{TeV}$, Phys. Rev. Lett. 119 (2017) 242301 [arXiv: 1709.05260] [INSPIRE].

[40] ALICE collaboration, Study of $\mathrm{J} / \psi$ azimuthal anisotropy at forward rapidity in $\mathrm{Pb}-\mathrm{Pb}$ collisions at $\sqrt{s_{\mathrm{NN}}}=5.02 \mathrm{TeV}, \mathrm{JHEP} 02$ (2019) 012 [arXiv:1811.12727] [INSPIRE].

[41] ATLAS collaboration, Prompt and non-prompt $J / \psi$ elliptic flow in $\mathrm{Pb}+\mathrm{Pb}$ collisions at $\sqrt{s_{\mathrm{NN}}}=5.02 \mathrm{TeV}$ with the ATLAS detector, Eur. Phys. J. C 78 (2018) 784 [arXiv: 1807.05198] [INSPIRE].

[42] ALICE collaboration, The ALICE experiment at the CERN LHC, 2008 JINST 3 S08002 [INSPIRE].

[43] ALICE collaboration, Performance of the ALICE experiment at the CERN LHC, Int. J. Mod. Phys. A 29 (2014) 1430044 [arXiv:1402 .4476] [INSPIRE]. 
[44] ALICE collaboration, Alignment of the ALICE Inner Tracking System with cosmic-ray tracks, 2010 JINST 5 P03003 [arXiv:1001.0502] [INSPIRE].

[45] ALICE collaboration, Performance of the ALICE VZERO system, 2013 JINST 8 P10016 [arXiv: 1306.3130] [INSPIRE].

[46] ALICE collaboration, ALICE luminosity determination for $p$-Pb collisions at $\sqrt{s_{\mathrm{NN}}}=8.16$ $\mathrm{TeV}$, ALICE-PUBLIC-2018-002 (2018).

[47] ALICE collaboration, Pseudorapidity density of charged particles in $p+P b$ collisions at $\sqrt{s_{\mathrm{NN}}}=5.02 \mathrm{TeV}$, Phys. Rev. Lett. 110 (2013) 032301 [arXiv:1210.3615] [INSPIRE].

[48] ALICE collaboration, Charged-particle pseudorapidity density at mid-rapidity in $p$ - $\mathrm{Pb}$ collisions at $\sqrt{s_{\mathrm{NN}}}=8.16 \mathrm{TeV}$, Eur. Phys. J. C 79 (2019) 307 [arXiv:1812.01312] [INSPIRE].

[49] S. Roesler, R. Engel and J. Ranft, The Monte Carlo event generator DPMJET-III, in the proceedings of the International Conference on Advanced Monte Carlo for Radiation Physics, Particle Transport Simulation and Applications (MC 2000), October 23-26, Lisbon, Portugal (2000) [hep-ph/0012252] [INSPIRE].

[50] R. Brun et al., GEANT: detector description and simulation tool, CERN-W-5013 (1993).

[51] T. Pierog, I. Karpenko, J.M. Katzy, E. Yatsenko and K. Werner, EPOS LHC: test of collective hadronization with data measured at the CERN Large Hadron Collider, Phys. Rev. C 92 (2015) 034906 [arXiv: 1306.0121] [INSPIRE].

[52] ALICE collaboration, Quarkonium signal extraction in ALICE, ALICE-PUBLIC-2015-006 (2015).

[53] ALICE collaboration, Energy dependence of forward-rapidity $J / \psi$ and $\psi(2 S)$ production in pp collisions at the LHC, Eur. Phys. J. C $\mathbf{7 7}$ (2017) 392 [arXiv:1702.00557] [INSPIRE].

[54] ALICE collaboration, Inclusive quarkonium production at forward rapidity in pp collisions at $\sqrt{s}=8$ TeV, Eur. Phys. J. C 76 (2016) 184 [arXiv:1509.08258] [InSPIRE].

[55] D.J. Lange, The EvtGen particle decay simulation package, Nucl. Instrum. Meth. A 462 (2001) 152 [INSPIRE].

[56] E. Barberio and Z. Was, PHOTOS: a universal Monte Carlo for QED radiative corrections. Version 2.0, Comput. Phys. Commun. 79 (1994) 291 [InSPIRE].

[57] ALICE collaboration, Prompt and non-prompt $J / \psi$ production and nuclear modification at mid-rapidity in $p$ - $P b$ collisions at $\sqrt{s_{\mathrm{NN}}}=5.02 \mathrm{TeV}$, Eur. Phys. J. C 78 (2018) 466 [arXiv: 1802.00765] [INSPIRE].

[58] S.G. Weber, A. Dubla, A. Andronic and A. Morsch, Elucidating the multiplicity dependence of $J / \psi$ production in proton-proton collisions with PYTHIA8, Eur. Phys. J. C 79 (2019) 36 [arXiv: 1811.07744] [INSPIRE].

[59] LHCb collaboration, Measurement of $J / \psi$ production in pp collisions at $\sqrt{s}=7 \mathrm{TeV}$, Eur. Phys. J. C 71 (2011) 1645 [arXiv:1103.0423] [InSPIRE].

[60] H.J. Drescher, M. Hladik, S. Ostapchenko, T. Pierog and K. Werner, Parton based Gribov-Regge theory, Phys. Rept. 350 (2001) 93 [hep-ph/0007198] [InSPIRE].

[61] K. Werner, B. Guiot, I. Karpenko and T. Pierog, Analysing radial flow features in $p$ - $P b$ and p-p collisions at several TeV by studying identified particle production in EPOS3, Phys. Rev. C 89 (2014) 064903 [arXiv:1312.1233] [INSPIRE]. 
[62] ALICE collaboration, Studies of $J / \psi$ production at forward rapidity in $\mathrm{Pb}$ - $\mathrm{Pb}$ collisions at $\sqrt{s_{\mathrm{NN}}}=5.02 \mathrm{TeV}$, JHEP $02(2020) 041$ [arXiv: 1909.03158] [INSPIRE].

[63] ALICE collaboration, Centrality dependence of the charged-particle multiplicity density at midrapidity in $\mathrm{Pb}-\mathrm{Pb}$ collisions at $\sqrt{s_{\mathrm{NN}}}=5.02 \mathrm{TeV}$, Phys. Rev. Lett. 116 (2016) 222302 [arXiv: 1512.06104] [INSPIRE].

[64] ALICE collaboration, Measurement of an excess in the yield of $J / \psi$ at very low $p_{\mathrm{T}}$ in $\mathrm{Pb}-\mathrm{Pb}$ collisions at $\sqrt{s_{\mathrm{NN}}}=2.76 \mathrm{TeV}$, Phys. Rev. Lett. 116 (2016) 222301 [arXiv:1509.08802] [INSPIRE].

[65] ALICE collaboration, Coherent $J / \psi$ photoproduction at forward rapidity in ultra-peripheral Pb-Pb collisions at $\sqrt{s_{\mathrm{NN}}}=5.02 \mathrm{TeV}$, Phys. Lett. B 798 (2019) 134926 [arXiv:1904.06272] [INSPIRE].

[66] D. d'Enterria and A. Snigirev, Double, triple, and n-parton scatterings in high-energy proton and nuclear collisions, Adv. Ser. Direct. High Energy Phys. 29 (2018) 159

[arXiv: 1708.07519] [INSPIRE]. 


\section{The ALICE collaboration}

S. Acharya ${ }^{141}$, D. Adamová ${ }^{55}$, A. Adler ${ }^{74}$, J. Adolfsson ${ }^{81}$, M.M. Aggarwal ${ }^{100}$, G. Aglieri Rinella ${ }^{34}$, M. Agnello ${ }^{30}$, N. Agrawal ${ }^{10,54}$, Z. Ahammed ${ }^{141}$, S. Ahmad ${ }^{16}$, S.U. Ahn ${ }^{76}$, Z. Akbar ${ }^{51}$,

A. Akindinov ${ }^{92}$, M. Al-Turany ${ }^{107}$, S.N. Alam ${ }^{141}$, D.S.D. Albuquerque ${ }^{122}$, D. Aleksandrov ${ }^{88}$,

B. Alessandro ${ }^{59}$, H.M. Alfanda ${ }^{6}$, R. Alfaro Molina ${ }^{71}$, B. Ali ${ }^{16}$, Y. Ali $^{14}$, A. Alici ${ }^{10,26,54}$,

A. Alkin ${ }^{2,34}$, J. Alme ${ }^{21}$, T. Alt ${ }^{68}$, L. Altenkamper ${ }^{21}$, I. Altsybeev ${ }^{113}$, M.N. Anaam ${ }^{6}$, C. Andrei ${ }^{48}$,

D. Andreou ${ }^{34}$, H.A. Andrews ${ }^{111}$, A. Andronic ${ }^{144}$, M. Angeletti ${ }^{34}$, V. Anguelov ${ }^{104}$, C. Anson ${ }^{15}$,

T. Antičić ${ }^{108}$, F. Antinori ${ }^{57}$, P. Antonioli ${ }^{54}$, N. Apadula ${ }^{80}$, L. Aphecetche ${ }^{115}$, H. Appelshäuser ${ }^{68}$,

S. Arcelli ${ }^{26}$, R. Arnaldi ${ }^{59}$, M. Arratia ${ }^{80}$, I.C. Arsene ${ }^{20}$, M. Arslandok ${ }^{104}$, A. Augustinus ${ }^{34}$,

R. Averbeck ${ }^{107}$, S. Aziz ${ }^{78}$, M.D. Azmi ${ }^{16}$, A. Badalà ${ }^{56}$, Y.W. Baek ${ }^{41}$, S. Bagnasco $^{59}$, X. Bai $^{107}$,

R. Bailhache ${ }^{68}$, R. Bala ${ }^{101}$, A. Balbino ${ }^{30}$, A. Baldisseri ${ }^{137}$, M. Ball ${ }^{43}$, S. Balouza ${ }^{105}$, D. Banerjee ${ }^{3}$,

R. Barbera ${ }^{27}$, L. Barioglio ${ }^{25}$, G.G. Barnaföldi ${ }^{145}$, L.S. Barnby ${ }^{94}$, V. Barret ${ }^{134}$, P. Bartalini ${ }^{6}$,

K. Barth ${ }^{34}$, E. Bartsch ${ }^{68}$, F. Baruffaldi ${ }^{28}$, N. Bastid ${ }^{134}$, S. Basu ${ }^{143}$, G. Batigne ${ }^{115}$, B. Batyunya ${ }^{75}$, D. Bauri ${ }^{49}$, J.L. Bazo Alba ${ }^{112}$, I.G. Bearden ${ }^{89}$, C. Beattie ${ }^{146}$, C. Bedda ${ }^{63}$, N.K. Behera ${ }^{61}$, I. Belikov ${ }^{136}$, A.D.C. Bell Hechavarria ${ }^{144}$, F. Bellini ${ }^{34}$, R. Bellwied ${ }^{125}$, V. Belyaev ${ }^{93}$,

G. Bencedi ${ }^{145}$, S. Beole ${ }^{25}$, A. Bercuci ${ }^{48}$, Y. Berdnikov ${ }^{98}$, A. Berdnikova ${ }^{104}$, D. Berenyi ${ }^{145}$, R.A. Bertens ${ }^{130}$, D. Berzano ${ }^{59}$, M.G. Besoiu ${ }^{67}$, L. Betev ${ }^{34}$, A. Bhasin ${ }^{101}$, I.R. Bhat ${ }^{101}$, M.A. Bhat ${ }^{3}$, H. Bhatt ${ }^{49}$, B. Bhattacharjee ${ }^{42}$, A. Bianchi ${ }^{25}$, L. Bianchi ${ }^{25}$, N. Bianchi ${ }^{52}$, J. Bielčík ${ }^{37}$, J. Bielčíková ${ }^{95}$, A. Bilandzic ${ }^{105}$, G. Biro ${ }^{145}$, R. Biswas ${ }^{3}$, S. Biswas ${ }^{3}$, J.T. Blair ${ }^{119}$, D. Blau ${ }^{88}$, C. Blume ${ }^{68}$, G. Boca ${ }^{139}$, F. Bock ${ }^{96}$, A. Bogdanov ${ }^{93}$, S. Boi ${ }^{23}$, J. Bok ${ }^{61}$, L. Boldizsár ${ }^{145}$, A. Bolozdynya ${ }^{93}$, M. Bombara ${ }^{38}$, G. Bonomi ${ }^{140}$, H. Borel $^{137}$, A. Borissov $^{93}$, H. Bossi $^{146}$, E. Botta ${ }^{25}$, L. Bratrud ${ }^{68}$, P. Braun-Munzinger ${ }^{107}$, M. Bregant ${ }^{121}$, M. Broz ${ }^{37}$, E. Bruna ${ }^{59}$, G.E. Bruno ${ }^{106}$, M.D. Buckland ${ }^{127}$, D. Budnikov ${ }^{109}$, H. Buesching ${ }^{68}$, S. Bufalino ${ }^{30}$, O. Bugnon ${ }^{115}$, P. Buhler ${ }^{114}$, P. Buncic ${ }^{34}$, Z. Buthelezi ${ }^{72,131}$, J.B. Butt ${ }^{14}$, S.A. Bysiak ${ }^{118}$, D. Caffarri ${ }^{90}$,

A. Caliva ${ }^{107}$, E. Calvo Villar ${ }^{112}$, R.S. Camacho ${ }^{45}$, P. Camerini ${ }^{24}$, A.A. Capon ${ }^{114}$, F. Carnesecchi ${ }^{26}$,

R. Caron ${ }^{137}$, J. Castillo Castellanos ${ }^{137}$, A.J. Castro ${ }^{130}$, E.A.R. Casula ${ }^{55}$, F. Catalano ${ }^{30}$,

C. Ceballos Sanchez ${ }^{53}$, P. Chakraborty ${ }^{49}$, S. Chandra ${ }^{141}$, W. Chang ${ }^{6}$, S. Chapeland ${ }^{34}$,

M. Chartier ${ }^{127}$, S. Chattopadhyay ${ }^{141}$, S. Chattopadhyay ${ }^{110}$, A. Chauvin ${ }^{23}$, C. Cheshkov ${ }^{135}$,

B. Cheynis ${ }^{135}$, V. Chibante Barroso ${ }^{34}$, D.D. Chinellato ${ }^{122}$, S. Cho ${ }^{61}$, P. Chochula ${ }^{34}$,

T. Chowdhury ${ }^{134}$, P. Christakoglou ${ }^{90}$, C.H. Christensen ${ }^{89}$, P. Christiansen ${ }^{81}$, T. Chujo ${ }^{133}$,

C. Cicalo ${ }^{55}$, L. Cifarelli ${ }^{10,26}$, F. Cindolo ${ }^{54}$, G. Clai ${ }^{54}$, ii, J. Cleymans ${ }^{124}$, F. Colamaria ${ }^{53}$,

D. Colella ${ }^{53}$, A. Collu ${ }^{80}$, M. Colocci ${ }^{26}$, M. Concas ${ }^{59}$, iii, G. Conesa Balbastre ${ }^{79}$, Z. Conesa del

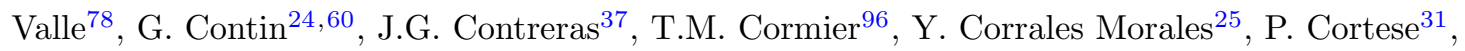

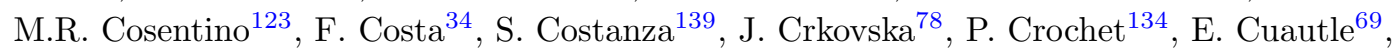
P. Cui ${ }^{6}$, L. Cunqueiro ${ }^{96}$, D. Dabrowski ${ }^{142}$, T. Dahms ${ }^{105}$, A. Dainese ${ }^{57}$, F.P.A. Damas ${ }^{115,137}$, M.C. Danisch ${ }^{104}$, A. Danu ${ }^{67}$, D. $\operatorname{Das}^{110}$, I. Das ${ }^{110}$, P. Das ${ }^{86}$, P. Das ${ }^{3}$, S. Das ${ }^{3}$, A. Dash ${ }^{86}$, S. $D_{a s h}^{49}$, S. De ${ }^{86}$, A. De Caro ${ }^{29}$, G. de Cataldo ${ }^{53}$, J. de Cuveland ${ }^{39}$, A. De Falco ${ }^{23}$, D. De Gruttola $^{10}$, N. De Marco ${ }^{59}$, S. De Pasquale ${ }^{29}$, S. Deb ${ }^{50}$, H.F. Degenhardt ${ }^{121}$, K.R. Deja ${ }^{142}$, A. Deloff ${ }^{85}$, S. Delsanto ${ }^{25,131}$, W. Deng ${ }^{6}$, D. Devetak ${ }^{107}$, P. Dhankher ${ }^{49}$, D. Di Bari ${ }^{33}$, A. Di

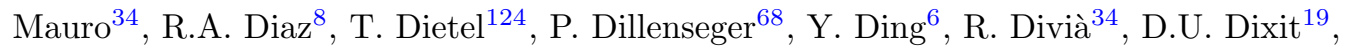
Ø. Djuvsland ${ }^{21}$, U. Dmitrieva ${ }^{62}$, A. Dobrin ${ }^{67}$, B. Dönigus ${ }^{68}$, O. Dordic ${ }^{20}$, A.K. Dubey ${ }^{141}$,

A. Dubla ${ }^{90,107}$, S. Dudi ${ }^{100}$, M. Dukhishyam ${ }^{86}$, P. Dupieux ${ }^{134}$, R.J. Ehlers ${ }^{96,146}$, V.N. Eikeland ${ }^{21}$,

D. Elia ${ }^{53}$, E. Epple ${ }^{146}$, B. Erazmus ${ }^{115}$, F. Erhardt ${ }^{99}$, A. Erokhin ${ }^{113}$, M.R. Ersdal ${ }^{21}$,

B. Espagnon ${ }^{78}$, G. Eulisse ${ }^{34}$, D. Evans ${ }^{111}$, S. Evdokimov ${ }^{91}$, L. Fabbietti ${ }^{105}$, M. Faggin ${ }^{28}$, J. Faivre ${ }^{79}$, F. Fan ${ }^{6}$, A. Fantoni ${ }^{52}$, M. Fasel ${ }^{96}$, P. Fecchio ${ }^{30}$, A. Feliciello ${ }^{59}$, G. Feofilov ${ }^{113}$, A. Fernández Téllez ${ }^{45}$, A. Ferrero ${ }^{137}$, A. Ferretti ${ }^{25}$, A. Festanti ${ }^{34}$, V.J.G. Feuillard ${ }^{104}$, J. Figiel ${ }^{118}$, S. Filchagin ${ }^{109}$, D. Finogeev ${ }^{62}$, F.M. Fionda ${ }^{21}$, G. Fiorenza ${ }^{53}$, F. Flor ${ }^{125}$, A.N. Flores ${ }^{119}$, 
S. Foertsch ${ }^{72}$, P. Foka ${ }^{107}$, S. Fokin ${ }^{88}$, E. Fragiacomo ${ }^{60}$, U. Frankenfeld ${ }^{107}$, U. Fuchs ${ }^{34}$, C. Furget ${ }^{79}$, A. Furs ${ }^{62}$, M. Fusco Girard ${ }^{29}$, J.J. Gaardhøje ${ }^{89}$, M. Gagliardi ${ }^{25}$, A.M. Gago ${ }^{112}$, A. Gal ${ }^{136}$, C.D. Galvan ${ }^{120}$, P. Ganoti ${ }^{84}$, C. Garabatos ${ }^{107}$, E. Garcia-Solis ${ }^{11}$, K. Garg ${ }^{115}$, C. Gargiulo ${ }^{34}$, A. Garibli ${ }^{87}$, K. Garner ${ }^{144}$, P. Gasik ${ }^{105,107}$, E.F. Gauger ${ }^{119}$, M.B. Gay Ducati ${ }^{70}$, M. Germain ${ }^{115}$, J. Ghosh ${ }^{110}$, P. Ghosh ${ }^{141}$, S.K. Ghosh ${ }^{3}$, M. Giacalone ${ }^{26}$, P. Gianotti ${ }^{52}$, P. Giubellino ${ }^{59,107}$, P. Giubilato ${ }^{28}$, P. Glässel ${ }^{104}$, A. Gomez Ramirez ${ }^{74}$, V. Gonzalez ${ }^{107,143}$, L.H. González-Trueba ${ }^{71}$, S. Gorbunov ${ }^{39}$, L. Görlich ${ }^{118}$, A. Goswami ${ }^{49}$, S. Gotovac ${ }^{35}$, V. Grabski ${ }^{71}$, L.K. Graczykowski ${ }^{142}$, K.L. Graham ${ }^{111}$, L. Greiner ${ }^{80}$, A. Grelli ${ }^{63}$, C. Grigoras $^{34}$, V. Grigoriev ${ }^{93}$, A. Grigoryan ${ }^{1}$, S. Grigoryan ${ }^{75}$, O.S. Groettvik ${ }^{21}$, F. Grosa ${ }^{30}$, J.F. Grosse-Oetringhaus ${ }^{34}$, R. Grosso ${ }^{107}$, R. Guernane ${ }^{79}$, M. Guittiere ${ }^{115}$, K. Gulbrandsen ${ }^{89}$, T. Gunji ${ }^{132}$, A. Gupta ${ }^{101}$, R. Gupta ${ }^{101}$, I.B. Guzman ${ }^{45}$, R. Haake ${ }^{146}$, M.K. Habib ${ }^{107}$, C. Hadjidakis ${ }^{78}$, H. Hamagaki ${ }^{82}$, G. Hamar ${ }^{145}$, M. Hamid ${ }^{6}$, R. Hannigan ${ }^{119}$, M.R. Haque ${ }^{63,86}$, A. Harlenderova ${ }^{107}$, J.W. Harris ${ }^{146}$, A. Harton ${ }^{11}$, J.A. Hasenbichler ${ }^{34}$, H. Hassan ${ }^{96}$, D. Hatzifotiadou ${ }^{10,54}$, P. Hauer ${ }^{43}$, L.B. Havener ${ }^{146}$, S. Hayashi ${ }^{132}$, S.T. Heckel ${ }^{105}$, E. Hellbär ${ }^{68}$, H. Helstrup ${ }^{36}$, A. Herghelegiu ${ }^{48}$, T. Herman ${ }^{37}$, E.G. Hernandez ${ }^{45}$, G. Herrera Corral ${ }^{9}$, F. Herrmann ${ }^{144}$, K.F. Hetland ${ }^{36}$, H. Hillemanns ${ }^{34}$, C. Hills ${ }^{127}$, B. Hippolyte ${ }^{136}$, B. Hohlweger ${ }^{105}$, J. Honermann ${ }^{144}$, D. Horak ${ }^{37}$, A. Hornung ${ }^{68}$, S. Hornung ${ }^{107}$, R. Hosokawa ${ }^{15}$, P. Hristov ${ }^{34}$, C. Huang ${ }^{78}$, C. Hughes ${ }^{130}$, P. Huhn ${ }^{68}$, T.J. Humanic ${ }^{97}$, H. Hushnud ${ }^{110}$, L.A. Husova ${ }^{144}$, N. Hussain ${ }^{42}$, S.A. Hussain ${ }^{14}$, D. Hutter ${ }^{39}$, J.P. Iddon ${ }^{34,127}$, R. Ilkaev ${ }^{109}$, H. Ilyas ${ }^{14}$, M. Inaba ${ }^{133}$, G.M. Innocenti ${ }^{34}$, M. Ippolitov ${ }^{88}$, A. Isakov ${ }^{95}$, M.S. Islam ${ }^{110}$, M. Ivanov ${ }^{107}$, V. Ivanov ${ }^{98}$, V. Izucheev ${ }^{91}$, B. Jacak ${ }^{80}$, N. Jacazio ${ }^{34}$, P.M. Jacobs ${ }^{80}$, S. Jadlovska ${ }^{117}$, J. Jadlovsky ${ }^{117}$, S. Jaelani ${ }^{63}$, C. Jahnke ${ }^{121}$, M.J. Jakubowska ${ }^{142}$, M.A. Janik ${ }^{142}$, T. Janson ${ }^{74}$, M. Jercic ${ }^{99}$, O. Jevons ${ }^{111}$, M. Jin ${ }^{125}$, F. Jonas ${ }^{96,144}$, P.G. Jones ${ }^{111}$, J. Jung ${ }^{68}$, M. Jung ${ }^{68}$, A. Jusko ${ }^{111}$, P. Kalinak ${ }^{64}$, A. Kalweit ${ }^{34}$, V. Kaplin ${ }^{93}$, S. Kar ${ }^{6}$, A. Karasu $\mathrm{Uysal}^{77}$, O. Karavichev ${ }^{62}$, T. Karavicheva ${ }^{62}$, P. Karczmarczyk ${ }^{34}$, E. Karpechev ${ }^{62}$, U. Kebschull ${ }^{74}$, R. Keidel ${ }^{47}$, M. Keil ${ }^{34}$, B. Ketzer ${ }^{43}$, Z. Khabanova ${ }^{90}$, A.M. Khan ${ }^{6}$, S. Khan ${ }^{16}$, S.A. Khan ${ }^{141}$,

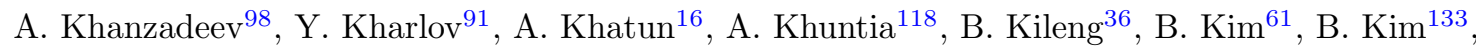
D. Kim ${ }^{147}$, D.J. Kim ${ }^{126}$, E.J. Kim ${ }^{73}$, H. Kim ${ }^{17}$, J. Kim${ }^{147}$, J.S. Kim${ }^{41}$, J. Kim ${ }^{104}$, J. Kim ${ }^{147}$,

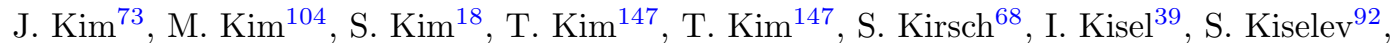
A. Kisiel ${ }^{142}$, J.L. Klay ${ }^{5}$, C. Klein ${ }^{68}$, J. Klein ${ }^{34,59}$, S. Klein ${ }^{80}$, C. Klein-Bösing ${ }^{144}$, M. Kleiner ${ }^{68}$, A. Kluge ${ }^{34}$, M.L. Knichel ${ }^{34}$, A.G. Knospe ${ }^{125}$, C. Kobdaj ${ }^{116}$, M.K. Köhler ${ }^{104}$, T. Kollegger ${ }^{107}$, A. Kondratyev ${ }^{75}$, N. Kondratyeva ${ }^{93}$, E. Kondratyuk ${ }^{91}$, J. Konig ${ }^{68}$, S.A. Konigstorfer ${ }^{105}$, P.J. Konopka ${ }^{34}$, G. Kornakov ${ }^{142}$, L. Koska ${ }^{117}$, O. Kovalenko ${ }^{85}$, V. Kovalenko ${ }^{113}$, M. Kowalski ${ }^{118}$, I. Králik ${ }^{64}$, A. Kravčáková ${ }^{38}$, L. Kreis ${ }^{107}$, M. Krivda ${ }^{64,111}$, F. Krizek ${ }^{95}$, K. Krizkova Gajdosova ${ }^{37}$, M. Krüger ${ }^{68}$, E. Kryshen ${ }^{98}$, M. Krzewicki ${ }^{39}$, A.M. Kubera ${ }^{97}$, V. Kučera ${ }^{34,61}$, C. Kuhn ${ }^{136}$, P.G. Kuijer ${ }^{90}$, L. Kumar ${ }^{100}$, S. Kundu ${ }^{86}$, P. Kurashvili ${ }^{85}$, A. Kurepin ${ }^{62}$, A.B. Kurepin ${ }^{62}$, A. Kuryakin ${ }^{109}$, S. Kushpil ${ }^{95}$, J. Kvapil ${ }^{111}$, M.J. Kweon ${ }^{61}$, J.Y. Kwon ${ }^{61}$, Y. Kwon ${ }^{147}$, S.L. La Pointe $^{39}$, P. La Rocca ${ }^{27}$, Y.S. Lai ${ }^{80}$, R. Langoy ${ }^{129}$, K. Lapidus ${ }^{34}$, A. Lardeux ${ }^{20}$, P. Larionov ${ }^{52}$, E. Laudi ${ }^{34}$, R. Lavicka ${ }^{37}$, T. Lazareva ${ }^{113}$, R. Lea ${ }^{24}$, L. Leardini ${ }^{104}$, J. Lee ${ }^{133}$, S. Lee ${ }^{147}$, F. Lehas $^{90}$, S. Lehner ${ }^{114}$, J. Lehrbach ${ }^{39}$, R.C. Lemmon ${ }^{94}$, I. León Monzón ${ }^{120}$, E.D. Lesser ${ }^{19}$,

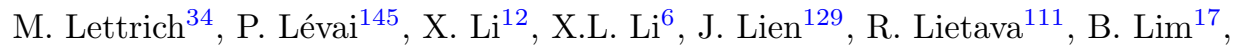
V. Lindenstruth ${ }^{39}$, A. Lindner ${ }^{48}$, S.W. Lindsay ${ }^{127}$, C. Lippmann ${ }^{107}$, M.A. Lisa ${ }^{97}$, A. Liu ${ }^{19}$,

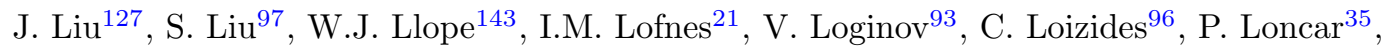
J.A. Lopez ${ }^{104}$, X. Lopez ${ }^{134}$, E. López Torres ${ }^{8}$, J.R. Luhder ${ }^{144}$, M. Lunardon ${ }^{28}$, G. Luparello ${ }^{60}$, Y.G. $\mathrm{Ma}^{40}$, A. Maevskaya ${ }^{62}$, M. Mager ${ }^{34}$, S.M. Mahmood ${ }^{20}$, T. Mahmoud ${ }^{43}$, A. Maire ${ }^{136}$, R.D. Majka ${ }^{146, \text { i , M. Malaev }}{ }^{98}$, Q.W. Malik ${ }^{20}$, L. Malinina ${ }^{75, \text { iv }}$, D. Mal'Kevich ${ }^{92}$, P. Malzacher ${ }^{107}$, G. Mandaglio ${ }^{32,56}$, V. Manko ${ }^{88}$, F. Manso $^{134}$, V. Manzari ${ }^{53}$, Y. Mao ${ }^{6}$, M. Marchisone ${ }^{135}$, J. Mareš ${ }^{66}$, G.V. Margagliotti ${ }^{24}$, A. Margotti ${ }^{54}$, J. Margutti ${ }^{63}$, A. Marín ${ }^{107}$, C. Markert $^{119}$, M. Marquard ${ }^{68}$, C.D. Martin $^{24}$, N.A. Martin ${ }^{104}$, P. Martinengo ${ }^{34}$, 
J.L. Martinez ${ }^{125}$, M.I. Martínez ${ }^{45}$, G. Martínez García ${ }^{115}$, S. Masciocchi ${ }^{107}$, M. Masera ${ }^{25}$, A. Masoni ${ }^{55}$, L. Massacrier ${ }^{78}$, E. Masson ${ }^{115}$, A. Mastroserio ${ }^{53,138}$, A.M. Mathis ${ }^{105}$, O. Matonoha ${ }^{81}$, P.F.T. Matuoka ${ }^{121}$, A. Matyja ${ }^{118}$, C. Mayer ${ }^{118}$, F. Mazzaschi ${ }^{25}$, M. Mazzilli53,

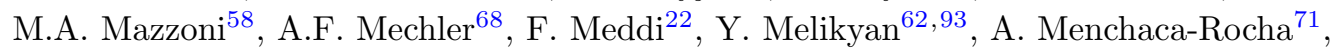
C. Mengke $^{6}$, E. Meninno ${ }^{29,114}$, M. Meres ${ }^{13}$, S. Mhlanga ${ }^{124}$, Y. Miake ${ }^{133}$, L. Micheletti ${ }^{25}$, L.C. Migliorin ${ }^{135}$, D.L. Mihaylov ${ }^{105}$, K. Mikhaylov ${ }^{75,92}$, A.N. Mishra ${ }^{69}$, D. Miśkowiec ${ }^{107}$, A. Modak ${ }^{3}$, N. Mohammadi ${ }^{34}$, A.P. Mohanty ${ }^{63}$, B. Mohanty ${ }^{86}$, M. Mohisin Khan ${ }^{16, v}$, Z. Moravcova ${ }^{89}$, C. Mordasini ${ }^{105}$, D.A. Moreira De Godoy ${ }^{144}$, L.A.P. Moreno ${ }^{45}$, I. Morozov ${ }^{62}$, A. Morsch ${ }^{34}$, T. Mrnjavac ${ }^{34}$, V. Muccifora ${ }^{52}$, E. Mudnic ${ }^{35}$, D. Mühlheim ${ }^{144}$, S. Muhuri ${ }^{141}$, J.D. Mulligan ${ }^{80}$, M.G. Munhoz ${ }^{121}$, R.H. Munzer ${ }^{68}$, H. Murakami ${ }^{132}$, S. Murray ${ }^{124}$, L. Musa ${ }^{34}$, J. Musinsky ${ }^{64}$, C.J. Myers ${ }^{125}$, J.W. Myrcha ${ }^{142}$, B. Naik ${ }^{49}$, R. Nair ${ }^{85}$, B.K. Nandi ${ }^{49}$, R. Nania ${ }^{10,54}$, E. Nappi ${ }^{53}$, M.U. Naru ${ }^{14}$, A.F. Nassirpour ${ }^{81}$, C. Nattrass ${ }^{130}$, R. Nayak ${ }^{49}$, T.K. Nayak ${ }^{86}$, S. Nazarenko ${ }^{109}$, A. Neagu ${ }^{20}$, R.A. Negrao De Oliveira ${ }^{68}$, L. Nellen ${ }^{69}$, S.V. Nesbo ${ }^{36}$, G. Neskovic ${ }^{39}$, D. Nesterov ${ }^{113}$, L.T. Neumann ${ }^{142}$, B.S. Nielsen ${ }^{89}$, S. Nikolaev ${ }^{88}$, S. Nikulin ${ }^{88}$, V. Nikulin ${ }^{98}$, F. Noferini ${ }^{10,54}$, P. Nomokonov ${ }^{75}$, J. Norman ${ }^{79,127}$, N. Novitzky ${ }^{133}$, P. Nowakowski ${ }^{142}$, A. Nyanin ${ }^{88}$, J. Nystrand ${ }^{21}$, M. Ogino ${ }^{82}$, A. Ohlson ${ }^{81,104}$, J. Oleniacz ${ }^{142}$, A.C. Oliveira Da Silva ${ }^{130}$, M.H. Oliver ${ }^{146}$, C. Oppedisano ${ }^{59}$, A. Ortiz Velasquez ${ }^{69}$,

A. Oskarsson ${ }^{81}$, J. Otwinowski ${ }^{118}$, K. Oyama ${ }^{82}$, Y. Pachmayer ${ }^{104}$, V. Pacik ${ }^{89}$, D. Pagano ${ }^{140}$, G. Paić ${ }^{69}$, J. Pan $^{143}$, S. Panebianco ${ }^{137}$, P. Pareek ${ }^{50,141}$, J. Park ${ }^{61}$, J.E. Parkkila ${ }^{126}$, S. Parmar ${ }^{100}$, S.P. Pathak ${ }^{125}$, B. Paul ${ }^{23}$, H. Pei ${ }^{6}$, T. Peitzmann ${ }^{63}$, X. Peng ${ }^{6}$, L.G. Pereira ${ }^{70}$, H. Pereira Da Costa $^{137}$, D. Peresunko ${ }^{88}$, G.M. Perez ${ }^{8}$, Y. Pestov ${ }^{4}$, V. Petráček ${ }^{37}$, M. Petrovici ${ }^{48}$, R.P. Pezzi ${ }^{70}$, S. Piano ${ }^{60}$, M. Pikna ${ }^{13}$, P. Pillot ${ }^{115}$, O. Pinazza ${ }^{34,54}$, L. Pinsky ${ }^{125}$, C. Pinto ${ }^{27}$, S. Pisano ${ }^{10,52}$, D. Pistone ${ }^{56}$, M. Płoskoń ${ }^{80}$, M. Planinic ${ }^{99}$, F. Pliquett ${ }^{68}$, M.G. Poghosyan ${ }^{96}$, B. Polichtchouk ${ }^{91}$, N. Poljak ${ }^{99}$, A. Pop ${ }^{48}$, S. Porteboeuf-Houssais ${ }^{134}$, V. Pozdniakov ${ }^{75}$, S.K. Prasad ${ }^{3}$, R. Preghenella ${ }^{54}$, F. Prino ${ }^{59}$, C.A. Pruneau ${ }^{143}$, I. Pshenichnov ${ }^{62}$, M. Puccio ${ }^{34}$, J. Putschke ${ }^{143}$, S. Qiu ${ }^{90}$, L. Quaglia ${ }^{25}$, R.E. Quishpe ${ }^{125}$, S. Ragoni ${ }^{111}$, S. Raha ${ }^{3}$, S. Rajput ${ }^{101}$, J. Rak ${ }^{126}$, A. Rakotozafindrabe ${ }^{137}$, L. Ramello ${ }^{31}$, F. Rami ${ }^{136}$, S.A.R. Ramirez ${ }^{45}$, R. Raniwala ${ }^{102}$, S. Raniwala ${ }^{102}$, S.S. Räsänen ${ }^{44}$, R. Rath ${ }^{50}$, V. Ratza ${ }^{43}$, I. Ravasenga ${ }^{90}$, K.F. Read ${ }^{96,130}$, A.R. Redelbach ${ }^{39}$, K. Redlich ${ }^{85}$, vi , A. Rehman ${ }^{21}$, P. Reichelt ${ }^{68}$, F. Reidt ${ }^{34}$, X. Ren ${ }^{6}$, R. Renfordt ${ }^{68}$, Z. Rescakova ${ }^{38}$, K. Reygers ${ }^{104}$, V. Riabov ${ }^{98}$, T. Richert ${ }^{81,89}$, M. Richter ${ }^{20}$, P. Riedler ${ }^{34}$, W. Riegler ${ }^{34}$, F. Riggi ${ }^{27}$, C. Ristea ${ }^{67}$, S.P. Rode ${ }^{50}$, M. Rodríguez Cahuantzi ${ }^{45}$, K. Røed ${ }^{20}$, R. Rogalev ${ }^{91}$, E. Rogochaya ${ }^{75}$, D. Rohr ${ }^{34}$, D. Röhrich ${ }^{21}$, P.S. Rokita ${ }^{142}$, F. Ronchetti ${ }^{52}$, A. Rosano ${ }^{56}$, E.D. Rosas ${ }^{69}$, K. Roslon ${ }^{142}$, A. Rossi ${ }^{28,57}$, A. Rotondi ${ }^{139}$, A. Roy ${ }^{50}$, P. Roy ${ }^{110}$, O.V. Rueda ${ }^{81}$, R. Rui ${ }^{24}$, B. Rumyantsev ${ }^{75}$, A. Rustamov ${ }^{87}$, E. Ryabinkin ${ }^{88}$, Y. Ryabov ${ }^{98}$, A. Rybicki ${ }^{118}$, H. Rytkonen ${ }^{126}$, O.A.M. Saarimaki ${ }^{44}$, S. Sadhu ${ }^{141}$, S. Sadovsky ${ }^{91}$, K. Šafař́k ${ }^{37}$, S.K. Saha ${ }^{141}$, B. Sahoo ${ }^{49}$, P. Sahoo ${ }^{49}$, R. Sahoo ${ }^{50}$, S. Sahoo ${ }^{65}$, P.K. Sahu ${ }^{65}$, J. Saini ${ }^{141}$, S. Sakai ${ }^{133}$, S. Sambyal ${ }^{101}$, V. Samsonov ${ }^{93,98}$, D. Sarkar ${ }^{143}$, N. Sarkar ${ }^{141}$, P. Sarma ${ }^{42}$, V.M. Sarti ${ }^{105}$, M.H.P. Sas ${ }^{63}$, E. Scapparone ${ }^{54}$, J. Schambach ${ }^{119}$, H.S. Scheid ${ }^{68}$, C. Schiaua ${ }^{48}$, R. Schicker ${ }^{104}$, A. Schmah ${ }^{104}$, C. Schmidt ${ }^{107}$, H.R. Schmidt ${ }^{103}$, M.O. Schmidt ${ }^{104}$, M. Schmidt ${ }^{103}$, N.V. Schmidt ${ }^{68,96}$, A.R. Schmier ${ }^{130}$, J. Schukraft ${ }^{89}$, Y. Schutz ${ }^{34,136}$, K. Schwarz ${ }^{107}$,

K. Schweda ${ }^{107}$, G. Scioli ${ }^{26}$, E. Scomparin ${ }^{59}$, J.E. Seger ${ }^{15}$, Y. Sekiguchi ${ }^{132}$, D. Sekihata ${ }^{132}$, I. Selyuzhenkov ${ }^{93,107}$, S. Senyukov ${ }^{136}$, D. Serebryakov ${ }^{62}$, A. Sevcenco ${ }^{67}$, A. Shabanov ${ }^{62}$, A. Shabetai ${ }^{115}$, R. Shahoyan ${ }^{34}$, W. Shaikh ${ }^{110}$, A. Shangaraev ${ }^{91}$, A. Sharma ${ }^{100}$, A. Sharma ${ }^{101}$, H. Sharma ${ }^{118}$, M. Sharma ${ }^{101}$, N. Sharma ${ }^{100}$, S. Sharma ${ }^{101}$, A.I. Sheikh ${ }^{141}$, K. Shigaki ${ }^{46}$, M. Shimomura ${ }^{83}$, S. Shirinkin ${ }^{92}$, Q. Shou ${ }^{40}$, Y. Sibiriak ${ }^{88}$, S. Siddhanta ${ }^{55}$, T. Siemiarczuk ${ }^{85}$, D. Silvermyr ${ }^{81}$, G. Simatovic ${ }^{90}$, G. Simonetti ${ }^{34}$, B. Singh ${ }^{105}$, R. Singh ${ }^{86}$, R. $\operatorname{Singh}^{101}$, R. $_{\text {Singh }}{ }^{50}$, V.K. Singh ${ }^{141}$, V. Singhal ${ }^{141}$, T. Sinha ${ }^{110}$, B. Sitar ${ }^{13}$, M. $\operatorname{Sitta}^{31}$, T.B. Skaali ${ }^{20}$, M. Slupecki ${ }^{44}$ N. Smirnov ${ }^{146}$, R.J.M. Snellings ${ }^{63}$, C. Soncco ${ }^{112}$, J. Song ${ }^{125}$, A. Songmoolnak ${ }^{116}$, F. Soramel ${ }^{28}$, 
S. Sorensen ${ }^{130}$, I. Sputowska ${ }^{118}$, J. Stachel ${ }^{104}$, I. Stan ${ }^{67}$, P.J. Steffanic ${ }^{130}$, E. Stenlund ${ }^{81}$, D. Stocco ${ }^{115}$, M.M. Storetvedt ${ }^{36}$, L.D. Stritto ${ }^{29}$, A.A.P. Suaide ${ }^{121}$, T. Sugitate ${ }^{46}$, C. Suire ${ }^{78}$, M. Suleymanov ${ }^{14}$, M. Suljic ${ }^{34}$, R. Sultanov ${ }^{92}$, M. Šumbera ${ }^{95}$, V. Sumberia ${ }^{101}$, S. Sumowidagdo ${ }^{51}$, S. Swain ${ }^{65}$, A. Szabo ${ }^{13}$, I. Szarka ${ }^{13}$, U. Tabassam ${ }^{14}$, S.F. Taghavi ${ }^{105}$, G. Taillepied ${ }^{134}$, J. Takahashi ${ }^{122}$, G.J. Tambave ${ }^{21}$, S. Tang ${ }^{6,134}$, M. Tarhini ${ }^{115}$, M.G. Tarzila ${ }^{48}$, A. Tauro ${ }^{34}$, G. Tejeda Muñoz ${ }^{45}$, A. Telesca ${ }^{34}$, L. Terlizzi ${ }^{25}$, C. Terrevoli ${ }^{125}$, D. Thakur ${ }^{50}$, S. Thakur ${ }^{141}$, D. Thomas ${ }^{119}$, F. Thoresen ${ }^{89}$, R. Tieulent ${ }^{135}$, A. Tikhonov ${ }^{62}$, A.R. Timmins ${ }^{125}$, A. Toia ${ }^{68}$,

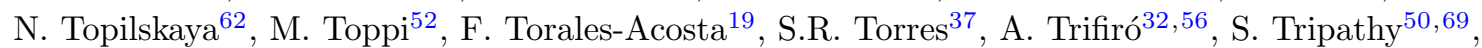

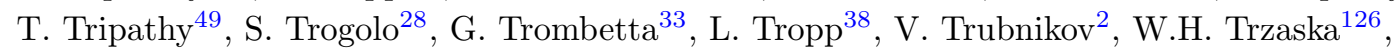
T.P. Trzcinski ${ }^{142}$, B.A. Trzeciak ${ }^{37,63}$, A. Tumkin ${ }^{109}$, R. Turrisi ${ }^{57}$, T.S. Tveter ${ }^{20}$, K. Ullaland $^{21}$, E.N. Umaka ${ }^{125}$, A. Uras ${ }^{135}$, G.L. Usai ${ }^{23}$, M. Vala ${ }^{38}$, N. Valle ${ }^{139}$, S. Vallero ${ }^{59}$, N. van der Kolk ${ }^{63}$, L.V.R. van Doremalen ${ }^{63}$, M. van Leeuwen ${ }^{63}$, P. Vande Vyvre ${ }^{34}$, D. Varga ${ }^{145}$, Z. Varga ${ }^{145}$, M. Varga-Kofarago ${ }^{145}$, A. Vargas ${ }^{45}$, M. Vasileiou ${ }^{84}$, A. Vasiliev ${ }^{88}$, O. Vázquez Doce ${ }^{105}$, V. Vechernin ${ }^{113}$, E. Vercellin ${ }^{25}$, S. Vergara Limón ${ }^{45}$, L. Vermunt ${ }^{63}$, R. Vernet ${ }^{7}$, R. Vértesi ${ }^{145}$, L. Vickovic ${ }^{35}$, Z. Vilakazi ${ }^{131}$, O. Villalobos Baillie ${ }^{111}$, G. Vino ${ }^{53}$, A. Vinogradov ${ }^{88}$, T. Virgili ${ }^{29}$, V. Vislavicius ${ }^{89}$, A. Vodopyanov ${ }^{75}$, B. Volkel ${ }^{34}$, M.A. Völkl ${ }^{103}$, K. Voloshin ${ }^{92}$, S.A. Voloshin ${ }^{143}$, G. Volpe ${ }^{33}$, B. von Haller ${ }^{34}$, I. Vorobyev ${ }^{105}$, D. Voscek ${ }^{117}$, J. Vrláková ${ }^{38}$, B. Wagner ${ }^{21}$, M. Weber ${ }^{114}$, A. Wegrzynek ${ }^{34}$, S.C. Wenzel ${ }^{34}$, J.P. Wessels ${ }^{144}$, J. Wiechula ${ }^{68}$, J. Wikne ${ }^{20}$, G. Wilk ${ }^{85}$, J. Wilkinson ${ }^{10,54}$, G.A. Willems ${ }^{144}$, E. Willsher ${ }^{111}$, B. Windelband ${ }^{104}$, M. Winn ${ }^{137}$, W.E. Witt ${ }^{130}$, J.R. Wright ${ }^{119}$, Y. $\mathrm{Wu}^{128}$, R. Xu ${ }^{6}$, S. Yalcin ${ }^{77}$, Y. Yamaguchi ${ }^{46}$, K. Yamakawa ${ }^{46}$,

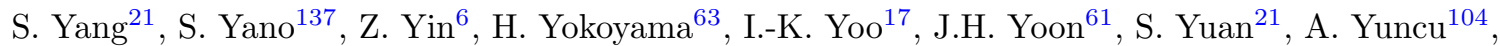
V. Yurchenko ${ }^{2}$, V. Zaccolo ${ }^{24}$, A. Zaman ${ }^{14}$, C. Zampolli ${ }^{34}$, H.J.C. Zanoli ${ }^{63}$, N. Zardoshti ${ }^{34}$,

A. Zarochentsev ${ }^{113}$, P. Závada ${ }^{66}$, N. Zaviyalov ${ }^{109}$, H. Zbroszczyk ${ }^{142}$, M. Zhalov ${ }^{98}$, S. Zhang ${ }^{40}$, X. Zhang ${ }^{6}$, Z. Zhang ${ }^{6}$, V. Zherebchevskii ${ }^{113}$, D. Zhou ${ }^{6}$, Y. Zhou ${ }^{89}$, Z. Zhou ${ }^{21}$, J. Zhu $^{6,107}$, Y. Zhu ${ }^{6}$, A. Zichichi ${ }^{10,26}$, G. Zinovjev ${ }^{2}$, N. Zurlo ${ }^{140}$

$i$ Deceased

ii Italian National Agency for New Technologies, Energy and Sustainable Economic Development (ENEA), Bologna, Italy

iii Dipartimento DET del Politecnico di Torino, Turin, Italy

iv M.V. Lomonosov Moscow State University, D.V. Skobeltsyn Institute of Nuclear, Physics, Moscow, Russia

$v$ Department of Applied Physics, Aligarh Muslim University, Aligarh, India

vi Institute of Theoretical Physics, University of Wroclaw, Poland

1 A.I. Alikhanyan National Science Laboratory (Yerevan Physics Institute) Foundation, Yerevan, Armenia

2 Bogolyubov Institute for Theoretical Physics, National Academy of Sciences of Ukraine, Kiev, Ukraine

3 Bose Institute, Department of Physics and Centre for Astroparticle Physics and Space Science (CAPSS), Kolkata, India

4 Budker Institute for Nuclear Physics, Novosibirsk, Russia

5 California Polytechnic State University, San Luis Obispo, California, United States

6 Central China Normal University, Wuhan, China

7 Centre de Calcul de l'IN2P3, Villeurbanne, Lyon, France

8 Centro de Aplicaciones Tecnológicas y Desarrollo Nuclear (CEADEN), Havana, Cuba

9 Centro de Investigación y de Estudios Avanzados (CINVESTAV), Mexico City and Mérida, Mexico

10 Centro Fermi - Museo Storico della Fisica e Centro Studi e Ricerche "Enrico Fermi', Rome, Italy

11 Chicago State University, Chicago, Illinois, United States 
13 Comenius University Bratislava, Faculty of Mathematics, Physics and Informatics, Bratislava, Slovakia

14 COMSATS University Islamabad, Islamabad, Pakistan

15 Creighton University, Omaha, Nebraska, United States

16 Department of Physics, Aligarh Muslim University, Aligarh, India

17 Department of Physics, Pusan National University, Pusan, Republic of Korea

18 Department of Physics, Sejong University, Seoul, Republic of Korea

19 Department of Physics, University of California, Berkeley, California, United States

20 Department of Physics, University of Oslo, Oslo, Norway

21 Department of Physics and Technology, University of Bergen, Bergen, Norway

22 Dipartimento di Fisica dell'Università 'La Sapienza' and Sezione INFN, Rome, Italy

23 Dipartimento di Fisica dell'Università and Sezione INFN, Cagliari, Italy

24 Dipartimento di Fisica dell'Università and Sezione INFN, Trieste, Italy

25 Dipartimento di Fisica dell'Università and Sezione INFN, Turin, Italy

26 Dipartimento di Fisica e Astronomia dell'Università and Sezione INFN, Bologna, Italy

27 Dipartimento di Fisica e Astronomia dell'Università and Sezione INFN, Catania, Italy

28 Dipartimento di Fisica e Astronomia dell'Università and Sezione INFN, Padova, Italy

29 Dipartimento di Fisica 'E.R. Caianiello' dell'Università and Gruppo Collegato INFN, Salerno, Italy

30 Dipartimento DISAT del Politecnico and Sezione INFN, Turin, Italy

31 Dipartimento di Scienze e Innovazione Tecnologica dell'Università del Piemonte Orientale and INFN Sezione di Torino, Alessandria, Italy

32 Dipartimento di Scienze MIFT, Università di Messina, Messina, Italy

33 Dipartimento Interateneo di Fisica 'M. Merlin' and Sezione INFN, Bari, Italy

34 European Organization for Nuclear Research (CERN), Geneva, Switzerland

35 Faculty of Electrical Engineering, Mechanical Engineering and Naval Architecture, University of Split, Split, Croatia

36 Faculty of Engineering and Science, Western Norway University of Applied Sciences, Bergen, Norway

37 Faculty of Nuclear Sciences and Physical Engineering, Czech Technical University in Prague, Prague, Czech Republic

38 Faculty of Science, P.J. S̆afárik University, Košice, Slovakia

39 Frankfurt Institute for Advanced Studies, Johann Wolfgang Goethe-Universität Frankfurt, Frankfurt, Germany

40 Fudan University, Shanghai, China

41 Gangneung-Wonju National University, Gangneung, Republic of Korea

42 Gauhati University, Department of Physics, Guwahati, India

43 Helmholtz-Institut für Strahlen- und Kernphysik, Rheinische Friedrich-Wilhelms-Universität Bonn, Bonn, Germany

44 Helsinki Institute of Physics (HIP), Helsinki, Finland

45 High Energy Physics Group, Universidad Autónoma de Puebla, Puebla, Mexico

46 Hiroshima University, Hiroshima, Japan

47 Hochschule Worms, Zentrum für Technologietransfer und Telekommunikation (ZTT), Worms, Germany

48 Horia Hulubei National Institute of Physics and Nuclear Engineering, Bucharest, Romania

49 Indian Institute of Technology Bombay (IIT), Mumbai, India

50 Indian Institute of Technology Indore, Indore, India

51 Indonesian Institute of Sciences, Jakarta, Indonesia

${ }^{52}$ INFN, Laboratori Nazionali di Frascati, Frascati, Italy

53 INFN, Sezione di Bari, Bari, Italy

54 INFN, Sezione di Bologna, Bologna, Italy

55 INFN, Sezione di Cagliari, Cagliari, Italy 
57 INFN, Sezione di Padova, Padova, Italy

58 INFN, Sezione di Roma, Rome, Italy

59 INFN, Sezione di Torino, Turin, Italy

60 INFN, Sezione di Trieste, Trieste, Italy

61 Inha University, Incheon, Republic of Korea

${ }^{62}$ Institute for Nuclear Research, Academy of Sciences, Moscow, Russia

${ }^{63}$ Institute for Subatomic Physics, Utrecht University/Nikhef, Utrecht, Netherlands

64 Institute of Experimental Physics, Slovak Academy of Sciences, Košice, Slovakia

${ }^{65}$ Institute of Physics, Homi Bhabha National Institute, Bhubaneswar, India

66 Institute of Physics of the Czech Academy of Sciences, Prague, Czech Republic

67 Institute of Space Science (ISS), Bucharest, Romania

${ }^{68}$ Institut für Kernphysik, Johann Wolfgang Goethe-Universität Frankfurt, Frankfurt, Germany

${ }^{69}$ Instituto de Ciencias Nucleares, Universidad Nacional Autónoma de México, Mexico City, Mexico

70 Instituto de Física, Universidade Federal do Rio Grande do Sul (UFRGS), Porto Alegre, Brazil

${ }^{71}$ Instituto de Física, Universidad Nacional Autónoma de México, Mexico City, Mexico

72 iThemba LABS, National Research Foundation, Somerset West, South Africa

73 Jeonbuk National University, Jeonju, Republic of Korea

74 Johann-Wolfgang-Goethe Universität Frankfurt Institut für Informatik, Fachbereich Informatik und Mathematik, Frankfurt, Germany

75 Joint Institute for Nuclear Research (JINR), Dubna, Russia

${ }^{76}$ Korea Institute of Science and Technology Information, Daejeon, Republic of Korea

77 KTO Karatay University, Konya, Turkey

78 Laboratoire de Physique des 2 Infinis, Irène Joliot-Curie, Orsay, France

79 Laboratoire de Physique Subatomique et de Cosmologie, Université Grenoble-Alpes, CNRS-IN2P3, Grenoble, France

80 Lawrence Berkeley National Laboratory, Berkeley, California, United States

81 Lund University Department of Physics, Division of Particle Physics, Lund, Sweden

82 Nagasaki Institute of Applied Science, Nagasaki, Japan

${ }^{83}$ Nara Women's University (NWU), Nara, Japan

84 National and Kapodistrian University of Athens, School of Science, Department of Physics, Athens, Greece

85 National Centre for Nuclear Research, Warsaw, Poland

${ }^{86}$ National Institute of Science Education and Research, Homi Bhabha National Institute, Jatni, India

87 National Nuclear Research Center, Baku, Azerbaijan

88 National Research Centre Kurchatov Institute, Moscow, Russia

89 Niels Bohr Institute, University of Copenhagen, Copenhagen, Denmark

${ }^{90}$ Nikhef, National institute for subatomic physics, Amsterdam, Netherlands

${ }^{91}$ NRC Kurchatov Institute IHEP, Protvino, Russia

${ }_{92}$ NRC "Kurchatov Institute" - ITEP, Moscow, Russia

93 NRNU Moscow Engineering Physics Institute, Moscow, Russia

94 Nuclear Physics Group, STFC Daresbury Laboratory, Daresbury, United Kingdom

95 Nuclear Physics Institute of the Czech Academy of Sciences, Řez u Prahy, Czech Republic

96 Oak Ridge National Laboratory, Oak Ridge, Tennessee, United States

97 Ohio State University, Columbus, Ohio, United States

98 Petersburg Nuclear Physics Institute, Gatchina, Russia

99 Physics department, Faculty of science, University of Zagreb, Zagreb, Croatia

100 Physics Department, Panjab University, Chandigarh, India

101 Physics Department, University of Jammu, Jammu, India

102 Physics Department, University of Rajasthan, Jaipur, India

103 Physikalisches Institut, Eberhard-Karls-Universität Tübingen, Tübingen, Germany

104 Physikalisches Institut, Ruprecht-Karls-Universität Heidelberg, Heidelberg, Germany 
105

106

107

108

109

110

111

112

113

115 SUBATECH, IMT Atlantique, Université de Nantes, CNRS-IN2P3, Nantes, France

116 Suranaree University of Technology, Nakhon Ratchasima, Thailand

117 Technical University of Košice, Košice, Slovakia

118 The Henryk Niewodniczanski Institute of Nuclear Physics, Polish Academy of Sciences, Cracow, Poland

119 The University of Texas at Austin, Austin, Texas, United States

120 Universidad Autónoma de Sinaloa, Culiacán, Mexico

121 Universidade de São Paulo (USP), São Paulo, Brazil

122 Universidade Estadual de Campinas (UNICAMP), Campinas, Brazil

123 Universidade Federal do ABC, Santo Andre, Brazil

124 University of Cape Town, Cape Town, South Africa

125 University of Houston, Houston, Texas, United States

126 University of Jyväskylä, Jyväskylä, Finland

127 University of Liverpool, Liverpool, United Kingdom

128 University of Science and Technology of China, Hefei, China

129 University of South-Eastern Norway, Tonsberg, Norway

130 University of Tennessee, Knoxville, Tennessee, United States

131 University of the Witwatersrand, Johannesburg, South Africa

132 University of Tokyo, Tokyo, Japan

133 University of Tsukuba, Tsukuba, Japan

134 Université Clermont Auvergne, CNRS/IN2P3, LPC, Clermont-Ferrand, France

135 Université de Lyon, Université Lyon 1, CNRS/IN2P3, IPN-Lyon, Villeurbanne, Lyon, France

136 Université de Strasbourg, CNRS, IPHC UMR 7178, F-67000 Strasbourg, France, Strasbourg, France

137 Université Paris-Saclay Centre d'Etudes de Saclay (CEA), IRFU, Départment de Physique Nucléaire $(D P h N)$, Saclay, France

138 Università degli Studi di Foggia, Foggia, Italy

139 Università degli Studi di Pavia, Pavia, Italy

140 Università di Brescia, Brescia, Italy

141 Variable Energy Cyclotron Centre, Homi Bhabha National Institute, Kolkata, India

142 Warsaw University of Technology, Warsaw, Poland

143 Wayne State University, Detroit, Michigan, United States

144 Westfälische Wilhelms-Universität Münster, Institut für Kernphysik, Münster, Germany

145 Wigner Research Centre for Physics, Budapest, Hungary

146 Yale University, New Haven, Connecticut, United States

147 Yonsei University, Seoul, Republic of Korea 